\title{
Convergence of an implicit Euler Galerkin scheme for Poisson-Maxwell-Stefan systems
}

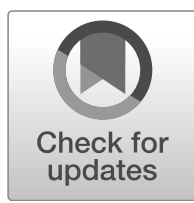

\section{Ansgar Jüngel ${ }^{1}$ (D) . Oliver Leingang ${ }^{1}$}

Received: 30 August 2018 / Accepted: 4 February 2019 /

Published online: 7 March 2019

(C) The Author(s) 2019

\begin{abstract}
A fully discrete Galerkin scheme for a thermodynamically consistent transient Maxwell-Stefan system for the mass particle densities, coupled to the Poisson equation for the electric potential, is investigated. The system models the diffusive dynamics of an isothermal ionized fluid mixture with vanishing barycentric velocity. The equations are studied in a bounded domain, and different molar masses are allowed. The Galerkin scheme preserves the total mass, the nonnegativity of the particle densities, their boundedness and satisfies the second law of thermodynamics in the sense that the discrete entropy production is nonnegative. The existence of solutions to the Galerkin scheme and the convergence of a subsequence to a solution to the continuous system is proved. Compared to previous works, the novelty consists in the treatment of the drift terms involving the electric field. Numerical experiments show the sensitive dependence of the particle densities and the equilibration rate on the molar masses.
\end{abstract}

Keywords Maxwell-Stefan systems · Cross diffusion · Ionized fluid mixtures · Entropy method · Finite-element approximation · Galerkin method . Numerical convergence

Mathematics Subject Classification (2010) $35 \mathrm{~K} 51 \cdot 35 \mathrm{~K} 55 \cdot 82 \mathrm{~B} 35$

Communicated by: Enrique Zuazua

The authors acknowledge partial support from the Austrian Science Fund (FWF), P27352, P30000, $\mathrm{F} 65$, and $\mathrm{W} 1245$

Ansgar Jüngel

juengel@tuwien.ac.at

Oliver Leingang

oliver.leingang@tuwien.ac.at

1 Institute for Analysis and Scientific Computing, Vienna University of Technology,

Wiedner Hauptstraße 8-10, 1040 Vienna, Austria 


\section{Introduction}

The Maxwell-Stefan equations describe the dynamics of a fluid mixture in the diffusive regime. They have numerous applications, for instance, in sedimentation, dialysis, electrolysis, and ion exchange. While Maxwell-Stefan models have been investigated since several decades from a modeling and simulation viewpoint in the engineering literature (e.g., [17]), the mathematical and numerical analysis started more recently $[1,20]$. The global existence of weak solutions under natural conditions was proved in $[8,26]$ for neutral mixtures. In the case of ion transport, the electric charges and the self-consistent electric potential need to be taken into account. To our knowledge, no mathematical results are available in the literature for such Poisson-Maxwell-Stefan models. In this paper, we prove the existence of a weak solution to a structure-preserving fully discrete Galerkin scheme and its convergence to the continuous problem. This provides, for the first time, a global existence result for Poisson-Maxwell-Stefan systems.

\subsection{Model equations}

We consider an ionized fluid mixture consisting of $n$ components with the partial mass density $\rho_{i}$, partial flux $J_{i}$, and molar mass $M_{i}$ of the $i$ th species. The evolution of the particle densities $\rho_{i}$ is governed by the partial mass balance equations

$$
\partial_{t} \rho_{i}+\operatorname{div} J_{i}=r_{i}(x), \quad i=1, \ldots, n,
$$

where $r_{i}$ are the production rates satisfying $\sum_{i=1}^{n} r_{i}(x)=0$ and $\sum_{i=1}^{n} J_{i}=0$. The variables are functions of the spatial variable $y$ and time $t$. The molar concentrations are defined by $c_{i}=\rho_{i} / M_{i}$ and $x_{i}=c_{i} / c_{\text {tot }}$ are the molar fractions, where $c_{\text {tot }}=\sum_{i=1}^{n} c_{i}$ denotes the total concentration and we have set $x=\left(x_{1}, \ldots, x_{n}\right)$. The partial fluxes $J_{i}$ and the gradients of the molar fractions $x_{i}$ are related by the (scaled) Maxwell-Stefan equations

$$
-\sum_{j=1}^{N} k_{i j}\left(\rho_{j} J_{i}-\rho_{i} J_{j}\right)=D_{i}:=\nabla x_{i}+\left(z_{i} x_{i}-(z \cdot x) \rho_{i}\right) \nabla \Phi, \quad i=1, \ldots, n .
$$

where $k_{i j}=k_{j i}$ are the rescaled (reciprocal) Maxwell-Stefan diffusivities, $D_{i}$ is the driving force, $z_{i}$ the electric charge of the $i$ th component, and $\Phi$ the electric potential. We refer to Section 2 for details on the modeling. These equations are coupled to the (scaled) Poisson equation

$$
-\lambda \Delta \Phi=\sum_{i=1}^{n} z_{i} c_{i}+f(y)
$$

where $\lambda$ is the scaled permittivity, and $f(y)$ is a fixed background charge. The equations are solved in a bounded domain $\Omega \subset \mathbb{R}^{d}(d \geq 1)$ and supplemented by the boundary conditions

$$
\begin{aligned}
& J_{i} \cdot v=0 \quad \text { on } \partial \Omega, i=1, \ldots, n, \\
& \Phi=\Phi_{D} \quad \text { on } \Gamma_{\mathrm{D}}, \quad \nabla \Phi \cdot v=0 \quad \text { on } \Gamma_{\mathrm{N}},
\end{aligned}
$$


where $\Gamma_{\mathrm{D}}$ models the electric contacts, $\Gamma_{N}=\partial \Omega \backslash \Gamma_{\mathrm{D}}$ is the union of insulating boundary segments, and $v$ denotes the exterior unit normal vector to $\partial \Omega$. This means that the mixture cannot leave the container $\Omega$ and an electric field is applied at the contacts $\Gamma_{\mathrm{D}}$. The initial conditions are given by

$$
\rho_{i}(\cdot, 0)=\rho_{i}^{0} \quad \text { in } \Omega, \quad i=1, \ldots, n .
$$

We assume that the total mass is constant initially, $\sum_{i=1}^{n} \rho_{i}^{0}=1$, which implies from (1) that the total mass is constant for all times, $\sum_{i=1}^{n} \rho_{i}(t)=1$, expressing total mass conservation.

Observe that (2) defines a linear system in the diffusion fluxes. Since $\sum_{i=1}^{n} D_{i}=$ 0 , the kernel of that system is nontrivial, and we need to invert the relation between the fluxes $J_{i}$ and the driving forces $D_{i}$ on the orthogonal component of the kernel. It was shown in [26, Section 2] that we can write (2) as $D^{\prime}=-A_{0} J^{\prime}$, where $D^{\prime}=$ $\left(D_{1}, \ldots, D_{n-1}\right), J^{\prime}=\left(J_{1}, \ldots, J_{n-1}\right)$, and $A_{0} \in \mathbb{R}^{(n-1) \times(n-1)}$ is invertible; see Section 3.1 for details. The $n$th components are recovered from $D_{n}=-\sum_{i=1}^{n-1} D_{i}$ and $J_{n}=-\sum_{i=1}^{n-1} J_{i}$. Thus, (1) can be written compactly as the cross-diffusion system $[1,26]$

$$
\partial_{t} \rho^{\prime}-\operatorname{div}\left(A_{0}^{-1} D^{\prime}\right)=r^{\prime}(x),
$$

where $\rho^{\prime}=\left(\rho_{1}, \ldots, \rho_{n-1}\right)$. However, $A_{0}^{-1}$ is not positive definite. To obtain a positive definite diffusion matrix, we need to transform the system. With the so-called entropy variables

$$
w_{i}=\frac{\log x_{i}}{M_{i}}-\frac{\log x_{n}}{M_{n}}+\left(\frac{z_{i}}{M_{i}}-\frac{z_{n}}{M_{n}}\right) \Phi, \quad i=1, \ldots, n-1,
$$

we may formulate (1) as

$$
\partial_{t} \rho^{\prime}-\operatorname{div}(B \nabla w)=r^{\prime}(x)
$$

where $B=\left(B_{i j}\right) \in \mathbb{R}^{(n-1) \times(n-1)}$ is symmetric and positive definite; see Section 3.1 for details. Here, $\rho^{\prime}$ and $x$ are interpreted as (invertible) functions of $w$ and $\Phi$. This transformation is well known in nonequilibrium thermodynamics, where $w_{i}$ is called the electro-chemical potential and $B$ is the mobility or Onsager matrix.

The transformation to entropy variables has two important advantages. First, introducing the entropy

$$
H(\rho)=\int_{\Omega} h(\rho) d y, \quad h(\rho)=c_{\text {tot }} \sum_{i=1}^{n} x_{i} \log x_{i}+\frac{\lambda}{2}\left|\nabla\left(\Phi-\Phi_{D}\right)\right|^{2},
$$

a formal computation shows that

$$
\frac{d H}{d t}+\int_{\Omega} \nabla w: B \nabla w d y=\int_{\Omega} \sum_{i=1}^{n} r_{i}(x) \frac{\partial h}{\partial \rho_{i}} d y,
$$

if $\Phi_{D}$ is constant and $\nabla w: B \nabla w=\sum_{i, j=1}^{n-1} B_{i j} \nabla w_{i} \cdot \nabla w_{j}$. (A discrete analog is shown in Theorem 1 below.) Thus, if the right-hand side is nonpositive, the entropy $t \mapsto H(\rho(t))$ is a Lyapunov functional and we may obtain suitable estimates for $w_{i}$. The entropy production (the diffusion term) is nonnegative, which expresses the second law of thermodynamics. This technique has been used in $[8,26]$ but without 
electric force terms. The derivation of gradient estimates is more delicate in the presence of the electric potential; see Lemma 8. Second, the densities $\rho_{i}=\rho_{i}(w)$ are automatically positive and bounded and it holds that $\sum_{i=1}^{n} \rho_{i}(w)=1$; see Corollary 7. This property is inherent of the transformation and it holds without the use of a maximum principle and independent of the functional setting.

The aim of this paper is to extend the global existence result of $[8,26]$ to MaxwellStefan systems with electric forces and to suggest a fully discrete Galerkin scheme that preserves the structure of the system, namely the nonnegativity of the particle densities, the $L^{\infty}$ bound $\sum_{i=1}^{n} \rho_{i}=1$, and a discrete analog of the entropy production inequality (10).

\subsection{State of the art}

Before presenting our main results, we briefly review the state of the art of MaxwellStefan models. They were already derived in the nineteenth century by Maxwell using kinetic gas theory [30] and Stefan using continuum mechanics [37]. A more mathematical derivation from the Boltzmann equation can be found in [4, 19], including a non-isothermal setting [22]. An advantage of the Maxwell-Stefan approach is that the definition of the driving forces can be adapted to the present physical situation, leading to very general and thermodynamically consistent models [2].

When electrolytes are considered, we need to take into account the electric force. Usually, this is done in the context of Nernst-Planck models $[32,34]$, where the diffusion flux $J_{i}$ only depends on the density gradient of the $i$ th component, thus without any cross-diffusion effects. Duncan and Toor [15] showed that cross-diffusion terms need to be taken into account in a ternary gas. Dreyer et al. [14] outline some deficiencies of Nernst-Planck models and propose thermodynamically consistent Maxwell-Stefan type models. A numerical comparison between Nernst-Planck and Maxwell-Stefan models can be found in [35].

The first global-in-time existence result to the Maxwell-Stefan equations (1)-(2) without Poisson equation was proved by Giovangigli and Massot [20] for initial data around the constant equilibrium state. The local-in-time existence of classical solutions was shown by Bothe [1]. The entropy structure of the Maxwell-Stefan system was revealed in [26], and a general global existence theorem could be shown. Further global existence results can be found in [21, 29]. The Maxwell-Stefan system was coupled to the heat equation [23] and to the incompressible Navier-Stokes equations [8]. In [19, Theorem 9.7.4] and [21, Theorem 4.3], the large-time asymptotics for initial data close to equilibrium was analyzed. The convergence to equilibrium for any initial data was investigated in [8, 26] without production terms and in [9] with production terms for reversible reactions. Salvarani and Soares proved a relaxation limit of the Maxwell-Stefan system to a system of linear heat equations [36].

Surprisingly, there are not many papers concerned with numerical schemes which preserve the properties of the solution like conservation of total mass, nonnegativity, and entropy production. Many approximation schemes can be found in the engineering literature, for instance finite-difference [27, 28] or finite-element [6] discretizations. In the mathematical literature, finite-volume [33] and mixed finiteelement [31] schemes as well as explicit finite-difference schemes with fast solvers 
[18] were proposed. The existence of discrete solutions was shown in [31], but only for ternary systems and under restrictions on the diffusion coefficients. The schemes of $[3,33]$ conserve the total mass, while those of $[3,11]$ also preserve the $L^{\infty}$ bounds. The result of [11] is based on maximum principle arguments. Note that we are able to show the $L^{\infty}$ bounds without the use of a maximum principle, as a result of the formulation in terms of entropy variables, and that we do not impose any restrictions on the diffusivities (except positivity).

All the cited results are concerned with the Maxwell-Stefan equations for neutral fluids, i.e., without electric effects. In this paper, we analyze for the first time Poisson-Maxwell-Stefan systems and show a discrete entropy production inequality. The cross-diffusion terms cause some mathematical difficulties which are not present in Nernst-Planck models.

\subsection{Main results}

Set $H_{D}^{1}(\Omega)=\left\{u \in H^{1}(\Omega): u=0\right.$ on $\left.\Gamma_{\mathrm{D}}\right\}$ and let $\left(\theta^{(k)}\right)$ be a basis of $H_{D}^{1}(\Omega)$ and $\left(v^{(k)}\right)$ be a basis of $H^{1}\left(\Omega ; \mathbb{R}^{n-1}\right)$ such that $v^{(k)} \in L^{\infty}\left(\Omega ; \mathbb{R}^{n-1}\right)$. We introduce the Galerkin spaces

$$
P_{N}=\operatorname{span}\left\{\theta^{(1)}, \ldots, \theta^{(N)}\right\}, \quad V_{N}=\operatorname{span}\left\{v^{(1)}, \ldots, v^{(N)}\right\} .
$$

Furthermore, let $T>0$ and $N \in \mathbb{N}$ and set $\tau=T / N>0$. We impose the following assumptions:

(A1) Domain: $\Omega \subset \mathbb{R}^{d}$ is a bounded domain with Lipschitz boundary $\partial \Omega=\Gamma_{\mathrm{D}} \cup$ $\Gamma_{\mathrm{N}}$, where $\Gamma_{\mathrm{D}} \cap \Gamma_{\mathrm{N}}=\emptyset, \Gamma_{\mathrm{N}}$ is open in $\partial \Omega$, and meas $\left(\Gamma_{\mathrm{D}}\right)>0$.

(A2) Given functions: The initial datum $\rho^{0}=\left(\rho_{1}^{0}, \ldots, \rho_{n}^{0}\right)$ is nonnegative and measurable, satisfying $\rho_{n}^{0}=1-\sum_{i=1}^{n-1} \rho_{i}^{0} \geq 0$. The boundary data $\Phi_{D} \in$ $H^{1}(\Omega) \cap L^{\infty}(\Omega)$ solves $-\lambda \Delta \Phi_{D}=f$ in $\Omega$ and $\nabla \Phi_{D} \cdot v=0$ on $\Gamma_{\mathrm{N}}$. Furthermore, let $f \in L^{\infty}(\Omega)$.

(A3) Diffusion matrix: For any given $\rho \in[0, \infty)^{n}$ satisfying $\sum_{i=1}^{n} \rho_{i}=1$, the transpose of the matrix $A=\left(A_{i j}\right) \in \mathbb{R}^{n \times n}$, defined by

$$
A_{i j}= \begin{cases}\sum_{\ell=1, \ell \neq i}^{n} k_{i \ell} \rho_{\ell} & \text { for } i=j, \\ -k_{i j} \rho_{i} & \text { for } i \neq j,\end{cases}
$$

has the kernel $\operatorname{ker}\left(A^{\top}\right)=\operatorname{span}\{1\}$, where $1=(1, \ldots, 1) \in \mathbb{R}^{n}$.

(A4) Production rates: The functions $r_{i} \in C^{0}\left([0,1]^{n} ; \mathbb{R}\right)$ satisfy $\sum_{i=1}^{n} r_{i}(x) \log x_{i} / M_{i} \leq C_{r}$ for some constant $C_{r}>0$ and all $x \in(0,1]^{n}$, $i=1, \ldots, n$.

Assumptions (A1) and (A2) are rather natural. The condition $\rho_{i} \log \rho_{i} \in L^{1}(\Omega)$ is needed to apply the entropy method. By definition of $A$, it holds that $\operatorname{ker}\left(A^{\top}\right) \subset$ $\operatorname{span}\{1\}$. If $k_{i j}>0$ (and $\rho_{j}>0$ ), a computation shows that $\operatorname{span}\{1\}=\operatorname{ker}\left(A^{\top}\right)$. For the general case $k_{i j} \geq 0$, this property cannot be guaranteed and needs to be assumed. This explains Assumption (A3). Assumption (A4) is needed to derive the entropy production inequality (10). It is satisfied for reversible reactions, see [9, Lemma 6] or [10]. It also holds in the context of a tumor-growth model, see [25]. 
We consider the implicit Euler Galerkin scheme

$$
\begin{aligned}
& \frac{1}{\tau} \int_{\Omega}\left(\rho^{\prime}\left(u^{k}+w_{D}, \Phi^{k}\right)-\rho^{\prime}\left(u^{k-1}+w_{D}, \Phi^{k-1}\right)\right) \cdot \phi d y+\varepsilon \int_{\Omega} u^{k} \cdot \phi d y \\
& +\int_{\Omega} \nabla \phi: B\left(u^{k}+w_{D}, \Phi^{k}\right) \nabla\left(u^{k}+w_{D}\right) d y=\int_{\Omega} r^{\prime}\left(x\left(u^{k}+w_{D}, \Phi^{k}\right)\right) \cdot \phi d y, \\
& \lambda \int_{\Omega} \nabla \Phi^{k} \cdot \nabla \theta d y=\int_{\Omega}\left(\sum_{i=1}^{n} z_{i} c_{i}\left(u^{k}+w_{D}, \Phi^{k}\right)+f(y)\right) \theta d y
\end{aligned}
$$

for $\phi \in V_{N}, \theta \in P_{N}, \varepsilon>0$, and we have defined

$$
w_{D}=\left(w_{D, 1}, \ldots, w_{D, n-1}\right), \quad w_{D, i}=\left(\frac{z_{i}}{M_{i}}-\frac{z_{n}}{M_{n}}\right) \Phi_{D} .
$$

The discrete entropy variables are given by $w^{k}=u^{k}+w_{D}$, and we used the notation $c_{i}\left(w^{k}, \Phi^{k}\right)=\rho_{i}\left(w^{k}, \Phi^{k}\right) / M_{i}, x_{i}\left(w^{k}, \Phi^{k}\right)=c_{i}\left(w^{k}, \Phi^{k}\right) / c_{\text {tot }}^{k}$ for $i=1, \ldots, n$, and $c_{\text {tot }}^{k}=\sum_{i=1}^{n} \rho_{i}\left(w^{k}, \Phi^{k}\right) / M_{i}$.

Furthermore, let $\Phi^{0} \in H^{1}(\Omega) \cap L^{\infty}(\Omega)$ be the unique solution to

$$
-\lambda \Delta \Phi^{0}=\sum_{i=1}^{n} z_{i} \frac{\rho_{i}^{0}}{M_{i}}+f(y) \text { in } \Omega, \quad \nabla \Phi^{0} \cdot v=0 \text { on } \Gamma_{\mathrm{N}}, \quad \Phi^{0}=\Phi_{D} \text { on } \Gamma_{\mathrm{D}} .
$$

This defines $\left(\rho^{0}, \Phi^{0}\right)$.

Theorem 1 (Existence for the Galerkin scheme) Let Assumptions (A1)-(A4) hold. Then, there exists a weak solution $\left(w^{k}, \Phi^{k}\right) \in V_{N} \times P_{N}$ to (12)-(13) with $w^{k}=$ $u^{k}+w_{D}$, satisfying

- Preservation of $L^{\infty}$ bounds: $0<\rho_{i}^{k}<1$ for $i=1, \ldots, n$;

- Conservation of total mass: $\sum_{i=1}^{n} \rho_{i}^{k}=1$ in $\Omega$;

- Discrete entropy production inequality:

$$
\begin{aligned}
& H\left(\rho^{k}\right)+\tau \int_{\Omega} \nabla\left(w^{k}-w_{D}\right): B\left(w^{k}, \Phi^{k}\right) \nabla w^{k} d y+\varepsilon \tau \int_{\Omega}\left|w^{k}-w_{D}\right|^{2} d y \\
\leq & \tau C_{r}|\Omega|+\tau \int_{\Omega} \sum_{i=1}^{n} \frac{z_{i}}{M_{i}} r_{i}\left(x^{k}\right)\left(\Phi^{k}-\Phi_{D}\right) d y+H\left(\rho^{k-1}\right),
\end{aligned}
$$

where $\rho^{k}=\rho\left(w^{k}, \Phi^{k}\right)$.

Theorem 1 is proved by using a fixed-point argument in the entropy variables. Using $w^{k}-w_{D}$ as a test function in the fully discrete version of (8), we show in Section 4 that

$$
H\left(\rho^{k}\right)+\tau K \int_{\Omega} \sum_{i=1}^{n}\left|\nabla\left(x_{i}^{k}\right)^{1 / 2}\right|^{2} d y+\varepsilon \tau \int_{\Omega}\left|w^{k}-w_{D}\right|^{2} d y \leq \tau k K+H\left(\rho^{k-1}\right),
$$

where $K>0$ only depends on the given data. This is an estimated version of (10). The term involving $\varepsilon$ is needed to conclude a uniform $L^{2}$ estimate for $w^{k}$, which is sufficient to apply the Leray-Schauder fixed-point theorem in the finite-dimensional 
Galerkin space. The $\varepsilon$-independent gradient estimate for $x_{i}^{k}$ cannot be used since it does not give an estimate for $w_{i}^{k}$ (see (7)). It is possible to analyze system (12)-(13) for $\varepsilon=0$ - see Step 2 of the proof of Theorem 3-but we lose the information about $w^{k}$ and obtain a solution in terms of $\rho^{k}$. The term involving $\varepsilon$ is technical and not essential for the numerical simulations (or the structure preservation). However, we are not able to prove an existence result in terms of the entropy variable without such a regularization.

Remark 2 (Conservation of partial mass) When $r_{i}=0$, we have from (1) conservation of the partial mass $\left\|\rho_{i}\right\|_{L^{1}(\Omega)}$. This conservation property does not hold exactly on the discrete level because of the $\varepsilon$-regularization. It holds that for any $\delta>0$, there exists $\varepsilon_{0}>0$ such that for any $0<\varepsilon<\varepsilon_{0}(\varepsilon$ is the value in (12)),

$$
\begin{aligned}
\left|\left\|\rho_{i}^{k}\right\|_{L^{1}(\Omega)}-\left\|\rho_{i}^{0}\right\|_{L^{1}(\Omega)}\right| & \leq \delta\left\|\rho_{i}^{0}\right\|_{L^{1}(\Omega)}, \quad i=1, \ldots, n-1, \\
\left|\left\|\rho_{n}^{k}\right\|_{L^{1}(\Omega)}-\left\|\rho_{n}^{0}\right\|_{L^{1}(\Omega)}\right| & \leq \delta \sum_{i=1}^{n-1}\left\|\rho_{i}^{0}\right\|_{L^{1}(\Omega)} .
\end{aligned}
$$

The proof is the same as in [26, Theorem 4.1]. As $\delta>0$ can be chosen arbitrarily small, this shows that the numerical scheme preserves the partial mass approximately.

Theorem 3 (Convergence of the Galerkin solution) Let Assumptions (A1)-(A4) hold. Let $\left(\rho^{k}, \Phi^{k}\right)$ be a solution to (12)-(13) and set

$$
\rho_{i}^{\tau}(y, t)=\rho_{i}^{k}(y), \quad x_{i}^{\tau}(y, t)=x_{i}^{k}(y), \quad c_{i}^{\tau}(y, t)=c_{i}^{k}(y), \quad \Phi^{\tau}(y, t)=\Phi^{k}(y)
$$

for $y \in \Omega, t \in((k-1) \tau, k \tau], i=1, \ldots, n$ and introduce the shift operator $\left(\sigma_{\tau} \rho_{i}^{\tau}\right)(y, t)=\rho_{i}^{k-1}(y)$ for $y \in \Omega$ and $t \in((k-1) \tau, k \tau]$. Then, there exist subsequences (not relabeled) such that, in the subsequent limits $\varepsilon \rightarrow 0$, then $N \rightarrow \infty$, and finally $\tau \rightarrow 0$,

$$
\begin{aligned}
& \rho_{i}^{\tau} \rightarrow \rho_{i} \quad \text { strongly in } L^{p}\left(0, T ; L^{p}(\Omega)\right) \text { for any } p<\infty, \\
& x_{i}^{\tau} \rightarrow x_{i}, \quad \Phi^{\tau} \rightarrow \Phi \quad \text { weakly in } L^{2}\left(0, T ; H^{1}(\Omega)\right), \\
& \tau^{-1}\left(\rho_{i}^{\tau}-\sigma_{\tau}\left(\rho_{i}^{\tau}\right)\right) \rightarrow \partial_{t} \rho \quad \text { weakly in } L^{2}\left(0, T ; H^{1}(\Omega)^{\prime}\right), i=1, \ldots, n,
\end{aligned}
$$

and the limit $(\rho, \Phi)$ satisfies for all $\phi \in L^{2}\left(0, T ; H^{1}\left(\Omega ; \mathbb{R}^{n-1}\right)\right)$ and $\theta \in H_{D}^{1}(\Omega)$,

$$
\begin{aligned}
\int_{0}^{T}\left\langle\partial_{t} \rho^{\prime}, \phi\right\rangle d t+\int_{0}^{T} \int_{\Omega} \nabla \phi: & A_{0}^{-1}(\rho) D^{\prime} d y d t \\
\lambda \int_{\Omega} \nabla \Phi \cdot \nabla \theta d y & =\int_{0}^{T} \int_{\Omega} r^{\prime}\left(\sum_{i=1}^{n} z_{i} \frac{\rho_{i}}{M_{i}}+f(y)\right) \theta d y
\end{aligned}
$$

where $D_{i}=\nabla x_{i}+\left(z_{i} x_{i}-(z \cdot x) \rho_{i}\right) \nabla \Phi, \rho_{i}=c_{t o t} M_{i} x_{i}$, and $c_{\text {tot }}=\sum_{i=1}^{n} \rho_{i} / M_{i}$. Moreover, $\rho_{n}=1-\sum_{i=1}^{n-1} \rho_{i}$. 
In Theorem 3, $\langle\cdot, \cdot\rangle$ denotes the duality bracket between $H^{1}\left(\Omega ; \mathbb{R}^{n-1}\right)^{\prime}$ and $H^{1}\left(\Omega ; \mathbb{R}^{n-1}\right)$. The difficult part of the proof is the estimate of the diffusion term because of the contribution of the electric field. We show in Lemma 8 that

$$
\int_{\Omega} \nabla w^{k}: B \nabla w^{k} d y \geq K \int_{\Omega} \sum_{i=1}^{n} M_{i}^{1 / 2} \frac{\left|D_{i}^{k}\right|^{2}}{x_{i}^{k}} d y \geq K_{1} \int_{\Omega} \sum_{i=1}^{n}\left|\nabla\left(x_{i}^{k}\right)^{1 / 2}\right|^{2} d y-K_{2}
$$

holds for some constants $K, K_{1}, K_{2}>0$, which are independent of $\varepsilon, N$, and $\tau$. Then, the uniform $L^{\infty}$ bound for $x_{i}^{k}$ gives a uniform $H^{1}(\Omega)$ bound for $x_{i}^{k}$ and consequently for $\rho_{i}^{k}$. Weak compactness allows us to pass to the limits $\varepsilon \rightarrow 0$ and $N \rightarrow \infty$, and the limit $\tau \rightarrow 0$ is performed by means of the Aubin-Lions lemma.

The paper is organized as follows. In Section 2, we detail the thermodynamic modeling of system (1)-(3). Some auxiliary results on the formulation of the fluxes $J_{i}$ and the inversion of the map $\rho \mapsto w$ are presented in Section 3. Sections 4 and 5 are devoted to the proof of the main theorems. Finally, some numerical experiments are shown in Section 6.

\section{Modeling}

We consider an isothermal electrolytic mixture of $n$ fluid components in the bounded domain $\Omega \subset \mathbb{R}^{d}(d \geq 1)$ with boundary $\partial \Omega$. We assume that the mixture is not moving, so the barycentric velocity vanishes. The thermodynamic state of the mixture is described by the partial mass densities $\rho_{1}, \ldots, \rho_{n}$ and the electric field $E$. The partial mass density represents the mass of the substance per unit volume. In thermodynamics, one introduces also the molar concentrations $c_{i}$, signifying the amount of substance per unit volume. These quantities are related by $\rho_{i}=M_{i} c_{i}$, where $M_{i}$ is the molar mass, the mass of the substance, divided by its amount. The total concentration is defined by $c_{\text {tot }}=\sum_{i=1}^{n} c_{i}$. Furthermore, $x_{i}=c_{i} / c_{\text {tot }}=\rho_{i} /\left(c_{\mathrm{tot}} M_{i}\right)$ denotes the molar fraction, being the amount of the substance, divided by the total amount of all constituents of the mixture. We suppose the quasi-static approximation $E=-\nabla \Phi$, where $\Phi$ is the electric potential.

The evolution of the mass densities $\rho_{i}$ is governed by the partial mass balances $[13,(4)]$

$$
\partial_{t} \rho_{i}+\operatorname{div} J_{i}=r_{i}(x) \quad \text { in } \Omega, t>0, i=1, \ldots, n,
$$

where $x=\left(x_{1}, \ldots, x_{n}\right)$ is the vector of molar fractions, $J_{i}$ the diffusion flux, and $r_{i}(x)$ the mass production rate of the $i$ th species. We assume that the total flux and the total production vanish,

$$
\sum_{i=1}^{n} J_{i}=0, \quad \sum_{i=1}^{n} r_{i}(x)=0,
$$

which are necessary constraints to achieve total mass conservation, $\partial_{t} \sum_{i=1}^{n} \rho_{i}=0$. We suppose that the total initial mass is constant in space, $\sum_{i=1}^{n} \rho_{i}^{0}=\rho_{\text {tot }}>0$, which implies that the total mass is constant in space and time, $\sum_{i=1}^{n} \rho_{i}(t)=\rho_{\text {tot }}$ for $t>0$. 
The electric potential $\Phi$ is given by the Poisson equation [14, (3) and (25)]

$$
-\varepsilon_{0}(1+\chi) \Delta \Phi=F \sum_{i=1}^{n} z_{i} c_{i}+f(y) \text { in } \Omega,
$$

where $\varepsilon_{0}$ is the dielectric constant, $\chi$ the dielectric susceptibility, $F$ the Faraday constant, $z_{i}$ the charge number of the $i$ th species, and $f(y)$ with $y \in \Omega$ models the charge of fixed background ions.

The basic assumption of the Maxwell-Stefan theory is that the difference in speed and molar fractions leads to a diffusion flux. They are implicitly given by the driving forces $d_{i}$ according to [2, (200)]

$$
-\sum_{j=1}^{n} \frac{x_{j}\left(J_{i} / M_{i}\right)-x_{i}\left(J_{j} / M_{j}\right)}{c_{\text {tot }} D_{i j}}=d_{i}, \quad i=1, \ldots, n,
$$

where the numbers $D_{i j}=D_{j i}$ are the Maxwell-Stefan diffusivities. Inserting the definition $x_{i}=\rho_{i} /\left(c_{\text {tot }} M_{i}\right)$, we find that

$$
-\sum_{j=1}^{n} \frac{\rho_{j} J_{i}-\rho_{i} J_{j}}{c_{\mathrm{tot}}^{2} M_{i} M_{j} D_{i j}}=d_{i}
$$

In the present situation, the driving force is given by two components, the variation of the chemical potential $\mu_{i}$ and the contribution of the body forces $b_{i}$ [2, (211)]:

$$
d_{i}=\frac{c_{i} M_{i}}{R T} \nabla \mu_{i}-\frac{\rho_{i}}{R T}\left(b_{i}-b_{\mathrm{tot}}\right), \quad i=1, \ldots, n,
$$

where $R$ is the gas constant and $T$ the (constant) temperature. Since $\left(D_{i j}\right)$ is symmetric, summing (18) from $i=1, \ldots, n$ leads to $\sum_{i=1}^{n} d_{i}=0$. Furthermore, $\sum_{i=1}^{n} \nabla \mu_{i}$ vanishes too; see below. This shows that $b_{\text {tot }}=\rho_{\text {tot }}^{-1} \sum_{i=1}^{n} \rho_{i} b_{i}$. We assume that the only force is due to the electric field (i.e., we neglect effects of gravity), $b_{i}=-\left(z_{i} / M_{i}\right) F \nabla \Phi[35,(3)]$.

It remains to determine the chemical potential. We define it by $\mu_{i}=\partial h_{\text {mix }} / \partial \rho_{i}$, where $h_{\text {mix }}(\rho)=c_{\text {tot }} R T\left(\sum_{i=1}^{n} x_{i} \log x_{i}+1\right)$ is the mixing free energy density [13, (23)]. Then,

$$
\mu_{i}=\frac{1}{c_{\mathrm{tot}} M_{i}} \frac{\partial h_{\mathrm{mix}}}{\partial x_{i}}=\frac{R T}{M_{i}}\left(\log x_{i}+1\right),
$$

and the driving force becomes

$$
\begin{aligned}
d_{i} & =c_{i} \nabla \log x_{i}+\frac{\rho_{i} F}{R T}\left(\frac{z_{i}}{M_{i}}-\frac{1}{\rho_{\mathrm{tot}}} \sum_{j=1}^{n} \frac{z_{j} \rho_{j}}{M_{j}}\right) \nabla \Phi \\
& =c_{\text {tot }}\left(\nabla x_{i}+\frac{F}{R T}\left(z_{i} x_{i}-(z \cdot x) \frac{\rho_{i}}{\rho_{\text {tot }}}\right) \nabla \Phi\right),
\end{aligned}
$$

where $z=\left(z_{1}, \ldots, z_{n}\right)$ and $x=\left(x_{1}, \ldots, x_{n}\right)$. The Gibbs-Duhem equation

$$
\sum_{i=1}^{n} \rho_{i} \frac{\partial h_{\text {mix }}}{\partial \rho_{i}}-h_{\text {mix }}(\rho)=R T \sum_{i=1}^{n} \rho_{i} \frac{\log x_{i}+1}{M_{i}}-c_{\text {tot }} R T\left(\sum_{i=1}^{n} x_{i} \log x_{i}+1\right)=0
$$


shows that the pressure vanishes, which is consistent with our choice of the driving force (see $[2,(211)])$. The driving force in $[35,(7)]$ contains a non-vanishing pressure that is related to our expression for the total body force. The resulting driving force (19), however, is the same.

We summarize the model equations:

$$
\begin{aligned}
\partial_{t} \rho_{i}+\operatorname{div} J_{i} & =r_{i}(x), \quad i=1, \ldots, n, \\
-\varepsilon_{0}(1+\chi) \Delta \Phi & =F \sum_{i=1}^{n} z_{i} c_{i}+f(y), \\
-\sum_{j=1}^{n} \frac{\rho_{j} J_{i}-\rho_{i} J_{j}}{c_{\mathrm{tot}}^{3} M_{i} M_{j} D_{i j}} & =\frac{d_{i}}{c_{\mathrm{tot}}}=\nabla x_{i}+\frac{F}{R T}\left(z_{i} x_{i}-(z \cdot x) \frac{\rho_{i}}{\rho_{\mathrm{tot}}}\right) \nabla \Phi,
\end{aligned}
$$

and the relations

$$
c_{i}=\frac{\rho_{i}}{M_{i}}, \quad x_{i}=\frac{\rho_{i}}{c_{\mathrm{tot}} M_{i}}, \quad c_{\mathrm{tot}}=\sum_{i=1}^{n} c_{i} .
$$

Equations 1-(3) are obtained from (20)-(22) after setting $\lambda=\varepsilon_{0}(1+\chi) / F, k_{i j}=$ $1 /\left(c_{\text {tot }}^{3} M_{i} M_{j} D_{i j}\right)$, and $D_{i}=d_{i} / c_{\text {tot }}$ and after nondimensionalization. In particular, we scale the particle densities by $\rho_{\text {tot }}$ (then the scaled quantities satisfy $\sum_{i=1}^{n} \rho_{i}=1$ ) and the electric potential by $F /(R T)$.

\section{Auxiliary results}

We collect some auxiliary results needed for the existence analysis. The starting point is relation (2) between the fluxes $J_{i}$ and the gradients $\nabla x_{i}$. Observe that the coefficients $k_{i j}$ depend on $\rho_{i}$ via $c_{\text {tot }}=\sum_{i=1}^{n} \rho_{i} / M_{i}$. This dependency does not complicate the analysis since the results in Section 3 hold pointwise for any given $\rho_{i}$ and $c_{\text {tot }}$ is uniformly bounded by

$$
\frac{1}{\max _{i=1, \ldots, n} M_{i}} \leq c_{\text {tot }}=\sum_{i=1}^{n} \frac{\rho_{i}}{M_{i}} \leq \frac{1}{\min _{i=1, \ldots, n} M_{i}} .
$$

\subsection{Expressions for the diffusion fluxes}

We review three different expressions for the diffusion fluxes following $[8,26]$ and extend the formulas to electro-chemical potentials. We reformulate (2):

$$
D_{i}=-\sum_{j \neq i} k_{i j}\left(\rho_{j} J_{i}-\rho_{i} J_{j}\right)=-\sum_{j \neq i} k_{i j} \rho_{i} \rho_{j}\left(\frac{J_{i}}{\rho_{i}}-\frac{J_{j}}{\rho_{j}}\right) .
$$

The symmetry of $\left(k_{i j}\right)$ implies that $\sum_{i=1}^{n} D_{i}=0$. Compactly, we may write $D=$ $-A J$, where $D=\left(D_{1}, \ldots, D_{n}\right)^{\top}, J=\left(J_{1}, \ldots, J_{n}\right)^{\top}$, and $A=\left(A_{i j}\right)$ with

$$
A_{i j}= \begin{cases}\sum_{\ell=1, \ell \neq i}^{n} k_{i \ell} \rho_{\ell} & \text { for } i=j \\ -k_{i j} \rho_{i} & \text { for } i \neq j\end{cases}
$$


By Assumption (A3), it holds that $\operatorname{im}(A)=\operatorname{ker}\left(A^{\top}\right)^{\perp}=\operatorname{span}\{1\}^{\perp}$, where $1=$ $(1, \ldots, 1)^{\top} \in \mathbb{R}^{n}$. We conclude from [26, Lemma 2.2] that all eigenvalues of $\widetilde{A}:=\left.A\right|_{\operatorname{im}(A)}$ are positive uniformly in $\rho \in[0,1]^{n}$ and that $\widetilde{A}$ is invertible. Since $\sum_{i=1}^{n} J_{i}=0$, e $\underset{\sim}{A} J$ row of $J=\left(J_{1}, \ldots, J_{n}\right)$ is an element of $\operatorname{im}(A)$, so the linear system $D=-\widetilde{A} J$ can be inverted, yielding $J=-\widetilde{A}^{-1} D$.

We obtain another formulation by inverting the system in the first $n-1$ variables. Setting $D^{\prime}=\left(D_{1}, \ldots, D_{n-1}\right)$ and $J^{\prime}=\left(J_{1}, \ldots, J_{n-1}\right)$, we can write $D^{\prime}=-A_{0} J^{\prime}$, where the matrix $A_{0}=\left(A_{i j}^{0}\right) \in \mathbb{R}^{(n-1) \times(n-1)}$ is defined by

$$
A_{i j}^{0}= \begin{cases}\sum_{\ell=1, \ell \neq i}^{n-1}\left(k_{i \ell}-k_{i n}\right) \rho_{\ell}+k_{i n} & \text { if } i=j, \\ -\left(k_{i j}-k_{i n}\right) \rho_{i} & \text { if } i \neq j\end{cases}
$$

It is shown in [8, Lemma 4] that $A_{0}$ is invertible and $A_{0}^{-1}$ is bounded uniformly in $\rho \in[0,1]^{n}$. Thus, $J^{\prime}=-A_{0}^{-1} D^{\prime}$.

Finally, we invert the relations (23). Using $J_{n}=-\sum_{i=1}^{n-1} J_{i}$, these relations (or the equivalent form $D_{i}=-\sum_{j=1}^{n} A_{i j} J_{j}$ ) can be written as

$$
\frac{D_{i}}{\rho_{i}}-\frac{D_{n}}{\rho_{n}}=-\sum_{j=1}^{n-1} C_{i j} J_{j}
$$

where

$$
\begin{aligned}
C_{i j} & =\frac{A_{i j}}{\rho_{i}}-\frac{A_{i n}}{\rho_{i}}-\frac{A_{n j}}{\rho_{n}}+\frac{A_{n n}}{\rho_{n}}=\frac{Y_{i j}}{\rho_{i} \rho_{j}}-\frac{Y_{i n}}{\rho_{i} \rho_{n}}-\frac{Y_{n j}}{\rho_{n} \rho_{j}}+\frac{Y_{n n}}{\rho_{n}^{2}}, \\
Y_{i j} & = \begin{cases}\sum_{\ell=1, \ell \neq i}^{n} k_{i \ell} \rho_{i} \rho_{\ell} & \text { for } i=j, \\
-k_{i j} \rho_{i} \rho_{j} & \text { for } i \neq j .\end{cases}
\end{aligned}
$$

The matrix $-Y=\left(-Y_{i j}\right) \in \mathbb{R}^{n \times n}$ is symmetric (since $\left(k_{i j}\right)$ is symmetric), quasipositive, irreducible, and it has the strictly positive eigenvector 1 with eigenvalue zero. Hence, by the Perron-Frobenius theorem, the spectral bound of $\left(-Y_{i j}\right)$ is a simple eigenvalue (with value zero) and the spectrum of $\left(Y_{i j}\right)$ consists of numbers with positive real part and zero. Thus, $Y$ is positive semidefinite.

We claim that the matrix $C=\left(C_{i j}\right) \in \mathbb{R}^{(n-1) \times(n-1)}$ is positive definite on $\operatorname{span}\{1\}^{\perp}$. Indeed, let $y \in \operatorname{span}\{\rho\}^{\perp}$. Then, $y \cdot \rho=0$. Since $1 \cdot \rho=1$, we have $y \notin \operatorname{span}\{1\}=\operatorname{ker}(Y)$ and consequently, $\operatorname{span}\{\rho\}^{\perp} \subset \operatorname{ker}(Y)^{c}$. This means that $-Y$ is negative definite on $\operatorname{span}\{\rho\}^{\perp}$. A computation shows that for any vector $w=\left(w_{1}, \ldots, w_{n-1}\right) \in \mathbb{R}^{n-1}$, it holds that

$$
\sum_{i, j=1}^{n-1} C_{i j} w_{i} w_{j}=\sum_{i, j=1}^{n} \frac{Y_{i j}}{\rho_{i} \rho_{j}} \widetilde{w}_{i} \widetilde{w}_{j}
$$

where $\widetilde{w}_{i}=w_{i}$ for $i=1, \ldots, n-1$ and $\widetilde{w}_{n}=-\sum_{i=1}^{n-1} w_{i}$. Then, $\widetilde{w}=$ $\left(\widetilde{w}_{1}, \ldots, \widetilde{w}_{n}\right) \in \operatorname{span}\{1\}^{\perp}$. Since $-Y$ is negative definite on $\operatorname{span}\{\rho\}^{\perp}$, we infer that $\left(-Y_{i j} /\left(\rho_{i} \rho_{j}\right)\right)$ is negative definite on $\operatorname{span}\{1\}^{\perp}$. Therefore, $C$ is positive definite on $\operatorname{span}\{1\}^{\perp}$. Its inverse $B:=c_{\text {tot }} C^{-1}$ with $B=\left(B_{i j}\right)$ exists, only depends on the 
mass density vector $\rho$, and is positive definite uniformly for all $\rho \in[0,1]^{n}$ satisfying $\sum_{i=1}^{n} \rho_{i}=1[8$, Lemma 10]. We deduce from (25) and (2) that

$$
\begin{aligned}
J_{i} & =-\sum_{j=1}^{n-1} B_{i j}\left(\frac{D_{j}}{\rho_{j}}-\frac{D_{n}}{\rho_{n}}\right) \\
& =-\sum_{j=1}^{n-1} B_{i j}\left(\frac{\nabla \log x_{j}}{M_{j}}-\frac{\nabla \log x_{n}}{M_{n}}+\left(\frac{z_{j}}{M_{j}}-\frac{z_{n}}{M_{n}}\right) \nabla \Phi\right) \\
& =-\sum_{j=1}^{n-1} B_{i j} \nabla w_{j}
\end{aligned}
$$

for $i=1, \ldots, n-1$ and $J_{n}=-\sum_{i=1}^{n-1} J_{i}$, recalling definition (7) of $w_{i}$. We summarize:

Lemma 4 (Formulations of $J_{i}$ ) Eqs. 23 can be written equivalently as

$$
J=-\widetilde{A}^{-1} D, \quad J^{\prime}=-A_{0}^{-1} D^{\prime}, \quad J^{\prime}=-B \nabla w .
$$

The last expression for $J_{i}$ shows that the partial mass balances (1) can be formulated as

$$
\partial_{t} \rho^{\prime}-\operatorname{div}(B \nabla w)=r^{\prime}(\rho),
$$

where $\rho=\rho(w)$ and $B=B(\rho(w))$. By Definition (7), $w$ is a function of $\rho$ (and $\Phi)$. The inverse relation $\rho(w)$ is discussed in the following subsection.

\subsection{Inversion of $\rho \mapsto w$}

Definition (7) defines, for given $\Phi \in \mathbb{R}$, a mapping $x \mapsto w$. We claim that this mapping can be inverted. If the molar masses are all the same, $M:=M_{i}$, this can be done explicitly:

$$
\rho_{i}(w)=\frac{\exp \left(M w_{i}-\left(z_{i}-z_{n}\right) \Phi\right)}{1+\sum_{j=1}^{n-1} \exp \left(M w_{j}-\left(z_{j}-z_{n}\right) \Phi\right)}, \quad i=1, \ldots, n-1,
$$

and $\rho_{n}=1-\sum_{i=1}^{n-1} \rho_{i}$. Unfortunately, when the molar masses are different, we cannot derive an explicit formula. Instead, we adapt Lemma 6 in [8].

Lemma 5 (Inversion of $w$ and $x$ ) Let $\Phi \in \mathbb{R}$ and define the function

$$
W_{\Phi}:\left\{x=\left(x_{1}, \ldots, x_{n}\right) \in(0,1)^{n}: \sum_{i=1}^{n} x_{i}=1\right\} \rightarrow \mathbb{R}^{n-1}
$$

by $W_{\Phi}(x)=\left(w_{1}(x), \ldots, w_{n-1}(x)\right)$, where

$$
w_{i}(x)=\frac{\log x_{i}}{M_{i}}-\frac{\log x_{n}}{M_{n}}+\left(\frac{z_{i}}{M_{i}}-\frac{z_{n}}{M_{n}}\right) \Phi, \quad i=1, \ldots, n-1 .
$$


Then, $W_{\Phi}$ is invertible and we can define $x(w, \Phi)=\left(x^{\prime}(w, \Phi), x_{n}(w, \Phi)\right):=$ $W_{\Phi}^{-1}(w)$, where $x^{\prime}(w, \Phi)=\left(x_{1}, \ldots, x_{n-1}\right)$ and $x_{n}(w, \Phi):=1-\sum_{i=1}^{n-1} x_{i}$.

Proof The proof is similar to that one of [8, Lemma 6]. Let $w=\left(w_{1}, \ldots, w_{n-1}\right) \in$ $\mathbb{R}^{n-1}$ and $\Phi \in \mathbb{R}$ be given. Define the function $f:[0,1] \rightarrow[0, \infty)$ by

$$
f(s)=\sum_{i=1}^{n-1}(1-s)^{M_{i} / M_{n}} \exp \left[M_{i} w_{i}-M_{i}\left(\frac{z_{i}}{M_{i}}-\frac{z_{n}}{M_{n}}\right) \Phi\right], \quad s \in[0,1] .
$$

Then, $f$ is continuous, strictly decreasing, and $0=f(1)<f(s)<f(0)$ for $s \in$ $(0,1)$. Hence, there exists a unique fixed point $s_{0} \in(0,1)$ such that $f\left(s_{0}\right)=s_{0}$. We define

$$
x_{i}=\left(1-s_{0}\right)^{M_{i} / M_{n}} \exp \left[M_{i} w_{i}-M_{i}\left(\frac{z_{i}}{M_{i}}-\frac{z_{n}}{M_{n}}\right) \Phi\right]>0, \quad i=1, \ldots, n-1 .
$$

By definition, we have $\sum_{i=1}^{n-1} x_{i}=f\left(s_{0}\right)=s_{0}<1$. We set $x_{n}=1-s_{0}>0$ such that $\sum_{i=1}^{n} x_{i}=1$. Moreover, (28) can be written equivalently as

$$
\frac{\log x_{i}}{M_{i}}+\frac{\log \left(1-s_{0}\right)}{M_{n}}+\left(\frac{z_{i}}{M_{i}}-\frac{z_{n}}{M_{n}}\right) \Phi=w_{i},
$$

and since $1-s_{0}=x_{n}$, this shows that $W_{\Phi}^{-1}(w)=\left(x^{\prime}, x_{n}\right)$ is the inverse mapping.

Given $\rho \in[0,1]^{n}$, we know that $x_{i}=\rho_{i} /\left(c_{\text {tot }} M_{i}\right)$ for $i=1, \ldots, n$ and $\sum_{i=1}^{n} x_{i}=$ 1. This relation can be inverted too. We recall [8, Lemma 7]:

Lemma 6 (Inversion of $\rho$ and $x$ ) Let $x^{\prime} \in(0,1)^{n-1}$ and $x_{n}=1-\sum_{i=1}^{n-1} x_{i}>0$ be given and define for $i=1, \ldots, n$,

$$
\rho_{i}\left(x^{\prime}\right)=\rho_{i}:=c_{t o t} M_{i} x_{i}, \quad \text { where } c_{t o t}=\left(\sum_{j=1}^{n} M_{j} x_{j}\right)^{-1} .
$$

Then, $\rho=\left(\rho_{1}, \ldots, \rho_{n}\right)$ is the unique vector satisfying $\rho_{n}=1-\sum_{i=1}^{n-1} \rho_{i}>0$, $x_{i}=\rho_{i} /\left(c_{t o t} M_{i}\right)$ for $i=1, \ldots, n$, and $c_{\text {tot }}=\sum_{i=1}^{n} \rho_{i} / M_{i}$.

Combining Lemmas 5 and 6, we conclude as in [8] that the mapping $\rho \mapsto w$ can be inverted. In fact, we just have to define $\rho^{\prime}=\rho^{\prime}\left(x^{\prime}(w, \Phi)\right)$.

Corollary 7 (Inversion of $\rho$ and $w$ ) Let $w=\left(w_{1}, \ldots, w_{n-1}\right) \in \mathbb{R}^{n-1}$ and $\Phi \in \mathbb{R}$ be given. Then, there exists a unique vector $\rho=\left(\rho_{1}, \ldots, \rho_{n}\right) \in(0,1)^{n}$ satisfying $\sum_{i=1}^{n} \rho_{i}=1$ such that (7) holds for $\rho_{n}=1-\sum_{i=1}^{n-1} \rho_{i}$ and $x_{i}=\rho_{i} /\left(c_{t o t} M_{i}\right)$ with $c_{\text {tot }}=\sum_{i=1}^{n} \rho_{i} / M_{i}$. The mapping $\rho^{\prime}: \mathbb{R}^{n} \rightarrow(0,1)^{n-1}, \rho^{\prime}(w, \Phi)=\left(\rho_{1}, \ldots, \rho_{n-1}\right)$, is bounded, i.e., $\left|\rho^{\prime}(w, \Phi)\right| \leq 1$ for all $(w, \Phi) \in \mathbb{R}^{n}$. 


\section{Proof of theorem 1}

Step 1: existence of solutions. The idea is to apply the Leray-Schauder fixed-point theorem. We need to define the fixed-point operator. For this, let $\chi \in L^{\infty}\left(\Omega ; \mathbb{R}^{n-1}\right)$ and $\sigma \in[0,1]$. Since $(y, \Phi) \mapsto \rho_{i}(\omega(y), \Phi)$ is a bounded function with values in $(0,1)$, we can use Schauder's fixed-point theorem and standard arguments to show the existence of a solution $\Phi^{k}-\Phi_{D} \in P_{N}$ of the nonlinear finite-dimensional problem

$$
\lambda \int_{\Omega} \nabla \Phi^{k} \cdot \nabla \theta d y=\int_{\Omega}\left(\sum_{i=1}^{n} z_{i} c_{i}\left(\chi+w_{D}, \Phi^{k}\right)+f(y)\right) \theta d y
$$

for all $\theta \in P_{N}$. In particular, the solution is unique and we have $\Phi^{k} \in L^{\infty}(\Omega)$, for $\Phi \mapsto \rho_{i}(\omega, \Phi)$ is Lipschitz continuous and $\Phi_{D} \in L^{\infty}(\Omega)$.

Next, we wish to solve the linear finite-dimensional problem

$$
a(u, \phi)=\sigma F(\phi) \quad \text { for all } \phi \in V_{N},
$$

where

$$
\begin{aligned}
a(u, \phi)= & \int_{\Omega} \nabla \phi: B\left(\chi+w_{D}, \Phi^{k}\right) \nabla u d y+\varepsilon \int_{\Omega} u \cdot \phi d y \\
F(\phi)= & -\frac{1}{\tau} \int_{\Omega}\left(\rho^{\prime}\left(\chi+w_{D}, \Phi^{k}\right)-\rho^{\prime}\left(u^{k-1}+w_{D}, \Phi^{k-1}\right)\right) \cdot \phi d y \\
& +\int_{\Omega} r^{\prime}\left(x\left(\chi+w_{D}, \Phi^{k}\right)\right) \cdot \phi d y-\int_{\Omega} \nabla \phi: B\left(\chi+w_{D}, \Phi^{k}\right) \nabla w_{D} d y
\end{aligned}
$$

for $u, \phi \in V_{N}$, where we set in case $k=1, \rho^{\prime}\left(u^{0}+\omega_{D}\right):=\left(\rho^{0}\right)^{\prime}$. Since $\chi+w_{D} \in$ $L^{\infty}\left(\Omega ; \mathbb{R}^{n-1}\right)$ and $\Phi^{k} \in L^{\infty}(\Omega)$, Corollary 7 shows that $\rho\left(\chi+w_{D}, \Phi^{k}\right)$ is bounded. We know from Section 3.1 that the matrix $B=B\left(\chi+w_{D}, \Phi^{k}\right)$ is positive definite and its elements are bounded. We deduce that the forms $a$ and $F$ are continuous on $V_{N}$. Exploiting the equivalence of the norms in the finite-dimensional space $V_{N}$, we find that

$$
a(u, u) \geq \varepsilon\|u\|_{L^{2}(\Omega)}^{2} \geq \varepsilon K_{N}\|u\|_{H^{1}(\Omega)}^{2}
$$

for some constant $K_{N}>0$, which implies that $a$ is coercive on $V_{N}$. By the LaxMilgram lemma, there exists a unique solution $u \in V_{N} \subset L^{\infty}\left(\Omega ; \mathbb{R}^{n-1}\right)$ to (29) satisfying

$$
\varepsilon K_{N}\|u\|_{H^{1}(\Omega)}^{2} \leq a(u, u)=\sigma F(u) \leq K_{F}\|u\|_{H^{1}(\Omega)},
$$

for some constant $K_{F}$, which is independent of $\tau$ and $\sigma$. Using again the bounds for $\rho^{\prime}$ and $r^{\prime}$, we find that the constant $K_{F}$ is independent of $\Phi^{k}$. Since all norms are equivalent in the finite-dimensional setting, this provides a uniform $L^{\infty}(\Omega)$ bound for $u$. 
This defines the fixed-point operator $S: L^{\infty}\left(\Omega ; \mathbb{R}^{n-1}\right) \times[0,1] \rightarrow L^{\infty}\left(\Omega ; \mathbb{R}^{n-1}\right)$, $S(\chi, \sigma)=u$. Standard arguments, see for example [24], show that $S$ is continuous. Since $V_{N}$ is finite-dimensional, $S$ is also compact. Furthermore, $S(\chi, 0)=0$. Estimate (30) provides a uniform bound for all fixed points of $S(\cdot, \sigma)$. Thus, by the Leray-Schauder fixed-point theorem, there exists $u^{k} \in V_{N}$ such that $S\left(u^{k}, 1\right)=u^{k}$, and $w^{k}:=u^{k}+w_{D}, \Phi^{k}$ solve (12)-(13).

Step 2: proof of the discrete entropy production inequality (15). We use the test function $\tau\left(w^{k}-w_{D}\right) \in V_{N}$ in (12) and set $\rho^{k}:=\rho^{\prime}\left(w^{k}, \Phi^{k}\right)$ :

$$
\begin{aligned}
& \int_{\Omega}\left(\rho^{k}-\rho^{k-1}\right) \cdot\left(w^{k}-w_{D}\right) d y+\tau \int_{\Omega} \nabla\left(w^{k}-w_{D}\right): B\left(w^{k}, \Phi^{k}\right) \nabla w^{k} d y \\
& +\varepsilon \tau \int_{\Omega}\left|w^{k}-w_{D}\right|^{2} d y \leq \tau \int_{\Omega} r^{\prime}\left(x^{k}\right) \cdot\left(w^{k}-w_{D}\right) d y .
\end{aligned}
$$

We claim that the first term on the left-hand side is greater than the difference of the entropies at time steps $k$ and $k-1$. To show this, we split the entropy density into two parts, $h\left(\rho^{k}\right)=h_{1}\left(\rho^{k}\right)+h_{2}\left(\rho^{k}\right)$, where

$$
h_{1}\left(\rho^{k}\right)=c_{\text {tot }}^{k} \sum_{i=1}^{n} x_{i}^{k} \log x_{i}^{k}, \quad h_{2}\left(\Phi^{k}\right)=\frac{\lambda}{2}\left|\nabla\left(\Phi^{k}-\Phi_{D}\right)\right|^{2},
$$

where we recall that $x_{i}^{k}=\rho_{i}^{k} /\left(c_{\text {tot }}^{k} M_{i}\right)$ and $c_{\text {tot }}^{k}=\sum_{i=1}^{n} \rho_{i}^{k} / M_{i}$. By the convexity of $h_{1}$, we have

$$
h_{1}\left(\rho^{k}\right)-h_{1}\left(\rho^{k-1}\right) \leq \frac{\partial h_{1}}{\partial \rho^{\prime}}\left(\rho^{k}\right) \cdot\left(\rho^{k}-\rho^{k-1}\right)=\sum_{i=1}^{n}\left(\rho_{i}^{k}-\rho_{i}^{k-1}\right) \frac{\log x_{i}^{k}}{M_{i}} .
$$

Therefore, using $\rho_{n}^{k}-\rho_{n}^{k-1}=-\sum_{i=1}^{n-1}\left(\rho_{i}^{k}-\rho_{i}^{k-1}\right)$,

$$
\begin{aligned}
\int_{\Omega}\left(h_{1}\left(\rho^{k}\right)-h_{1}\left(\rho^{k-1}\right)\right) d x & \leq \int_{\Omega}\left(\sum_{i=1}^{n-1}\left(\rho_{i}^{k}-\rho_{i}^{k-1}\right) \frac{\log x_{i}^{k}}{M_{i}}+\left(\rho_{n}^{k}-\rho_{n}^{k-1}\right) \frac{\log x_{n}^{k}}{M_{n}}\right) d y \\
& =\int_{\Omega} \sum_{i=1}^{n-1}\left(\rho_{i}^{k}-\rho_{i}^{k-1}\right)\left(\frac{\log x_{i}^{k}}{M_{i}}-\frac{\log x_{n}^{k}}{M_{n}}\right) d y
\end{aligned}
$$

For the estimate of $h_{2}$, we first observe that

$$
\begin{aligned}
\sum_{i=1}^{n-1}\left(\rho_{i}^{k}-\rho_{i}^{k-1}\right)\left(\frac{z_{i}}{M_{i}}-\frac{z_{n}}{M_{n}}\right) & =\sum_{i=1}^{n-1}\left(\rho_{i}^{k}-\rho_{i}^{k-1}\right) \frac{z_{i}}{M_{i}}+\left(\rho_{n}^{k}-\rho_{n}^{k-1}\right) \frac{z_{n}}{M_{n}} \\
& =\sum_{n=1}^{n}\left(\rho_{i}^{k}-\rho_{i}^{k-1}\right) \frac{z_{i}}{M_{i}} .
\end{aligned}
$$


We infer from the Poisson Eq. 13 and Young's inequality that

$$
\begin{aligned}
& \int_{\Omega} \sum_{i=1}^{n-1}\left(\rho_{i}^{k}-\rho_{i}^{k-1}\right)\left(\frac{z_{i}}{M_{i}}-\frac{z_{n}}{M_{n}}\right)\left(\Phi^{k}-\Phi_{D}\right) d y \\
= & \int_{\Omega} \sum_{i=1}^{n}\left(\rho_{i}^{k}-\rho_{i}^{k-1}\right) \frac{z_{i}}{M_{i}}\left(\Phi^{k}-\Phi_{D}\right) d y=\int_{\Omega} \sum_{i=1}^{n} z_{i}\left(c_{i}^{k}-c_{i}^{k-1}\right)\left(\Phi^{k}-\Phi_{D}\right) d y \\
= & \lambda \int_{\Omega} \nabla\left(\left(\Phi^{k}-\Phi_{D}\right)-\left(\Phi^{k-1}-\Phi_{D}\right)\right) \cdot \nabla\left(\Phi^{k}-\Phi_{D}\right) d y \\
\geq & \frac{\lambda}{2} \int_{\Omega}\left|\nabla\left(\Phi^{k}-\Phi_{D}\right)\right|^{2} d y-\frac{\lambda}{2} \int_{\Omega}\left|\nabla\left(\Phi^{k-1}-\Phi_{D}\right)\right|^{2} d y \\
= & \int_{\Omega}\left(h_{2}\left(\Phi^{k}\right)-h_{2}\left(\Phi^{k-1}\right)\right) d y .
\end{aligned}
$$

Taking into account the property $r_{n}\left(\rho^{k}\right)=-\sum_{i=1}^{n-1} r_{i}\left(\rho^{k}\right)$, definition (7) of $w_{i}^{k}$, and Assumption (A4), we compute

$$
\begin{aligned}
f_{\Omega}^{\prime}\left(x^{k}\right) \cdot\left(w^{k}-w_{D}\right) d y= & \int_{\Omega} \sum_{i=1}^{n-1} r_{i}\left(x^{k}\right)\left(\frac{\log x_{i}^{k}}{M_{i}}-\frac{\log x_{n}^{k}}{M_{n}}\right) d y \\
& +\int_{\Omega} \sum_{i=1}^{n-1} r_{i}\left(x^{k}\right)\left(\frac{z_{i}}{M_{i}}-\frac{z_{n}}{M_{n}}\right)\left(\Phi^{k}-\Phi_{D}\right) d y \\
= & \int_{\Omega} \sum_{i=1}^{n} r_{i}\left(x^{k}\right) \frac{\log x_{i}^{k}}{M_{i}} d y+\int_{\Omega} \sum_{i=1}^{n} r_{i}\left(x^{k}\right) \frac{z_{i}}{M_{i}}\left(\Phi^{k}-\Phi_{D}\right) d y \\
\leq & C_{r}|\Omega|+\int_{\Omega} \sum_{i=1}^{n} r_{i}\left(x^{k}\right) \frac{z_{i}}{M_{i}}\left(\Phi^{k}-\Phi_{D}\right) d y
\end{aligned}
$$

Combining (31)-(33) gives the conclusion.

\section{Proof of theorem 3}

Let $\left(w^{k}, \Phi^{k}\right)$ be a weak solution to scheme (12)-(13) and define $\rho^{k}=\rho\left(w^{k}, \Phi^{k}\right)$.

Step 1: uniform estimates We derive estimates for $\rho^{k}$ and $\Phi^{k}$ independent of $\varepsilon, \tau$, and $N$. The starting point is the discrete entropy production inequality (15), and the main task is to estimate the diffusion part.

Lemma 8 (Estimate of the diffusion part) There exist constants $K_{1}>0$ and $K_{2}>0$, both independent of $\varepsilon, \tau$, and $N$, such that

$$
\int_{\Omega} \nabla\left(w^{k}-w_{D}\right): B \nabla w^{k} d y \geq K_{1} \sum_{i=1}^{n}\left\|\nabla\left(x_{i}^{k}\right)^{1 / 2}\right\|_{L^{2}(\Omega)}^{2}-K_{2} .
$$


Proof We drop the superindex $k$ in the proof to simplify the notation. Recall that $\widetilde{A}=\left.A\right|_{\operatorname{im}(A)}$, where $\operatorname{im}(\underset{\widetilde{A}}{A})=\operatorname{span}\{1\}^{\perp}$. We introduce as in the proof of Lemma 12 in [8] the symmetrization $\widetilde{A}_{S}=P^{-1 / 2} \widetilde{A} P^{1 / 2}$, where $P^{1 / 2}=M^{1 / 2} X^{1 / 2}$ and $M^{1 / 2}:=$ $\operatorname{diag}\left(M_{1}^{1 / 2}, \ldots, M_{n}^{1 / 2}\right), X^{1 / 2}:=\operatorname{diag}\left(x_{1}^{1 / 2}, \ldots, x_{n}^{1 / 2}\right)$. Then, $\widetilde{A}_{S}^{-1}=P^{-1 / 2} \widetilde{A}^{-1} P^{1 / 2}$ is a self-adjoint endomorphism whose smallest eigenvalue is bounded from below by some positive constant which depends only on $\left(k_{i j}\right)$.

Since $0=\sum_{i=1}^{n} J_{i}=\sum_{i=1}^{n}(B \nabla w)_{i}$, we can express the last component in terms of the other components, $(B \nabla w)_{n}=-\sum_{i=1}^{n-1}(B \nabla w)_{i}$, where $(B \nabla w)_{i}=$ $\sum_{j=1}^{n-1} B_{i j} \nabla w_{j}$ for $i=1, \ldots, n-1$. Then,

$$
\begin{aligned}
\nabla w: B \nabla w & =\sum_{i=1}^{n-1}\left\{\frac{\nabla \log x_{i}}{M_{i}}-\frac{\nabla \log x_{n}}{M_{n}}+\left(\frac{z_{i}}{M_{i}}-\frac{z_{n}}{M_{n}}\right) \nabla \Phi\right\} \cdot(B \nabla w)_{i} \\
& =\sum_{i=1}^{n-1} \frac{1}{M_{i}} \nabla\left(\log x_{i}+z_{i} \Phi\right) \cdot(B \nabla w)_{i}-\frac{1}{M_{n}} \nabla\left(\log x_{n}+z_{n} \Phi\right) \sum_{i=1}^{n-1}(B \nabla w)_{i} \\
& =\sum_{i=1}^{n} \frac{1}{M_{i}} \nabla\left(\log x_{i}+z_{i} \Phi\right) \cdot(B \nabla w)_{i}
\end{aligned}
$$

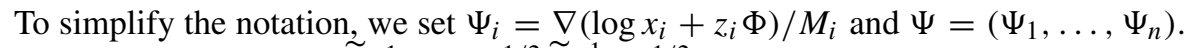
By Lemma $4, B \nabla w=\widetilde{A}^{-1} D=P^{1 / 2} \widetilde{A}_{S}^{-1} P^{-1 / 2} D$. Hence,

$$
\begin{aligned}
\nabla w: B \nabla w= & \Psi: B \nabla w=\Psi: M^{1 / 2} X^{1 / 2} \widetilde{A}_{S}^{-1} X^{-1 / 2} M^{-1 / 2} D \\
= & \sum_{i, j=1}^{n} \Psi_{i} M_{i}^{1 / 2} x_{i}^{1 / 2}\left(\widetilde{A}_{S}^{-1}\right)_{i j} x_{j}^{-1 / 2} M_{j}^{-1 / 2} D_{i} \\
= & \sum_{i, j=1}^{n}\left(2 \nabla x_{i}^{1 / 2}+z_{i} x_{i}^{1 / 2} \nabla \Phi\right) M_{i}^{-1 / 2}\left(\widetilde{A}_{S}^{-1}\right)_{i j} M_{j}^{-1 / 2} \\
& \times\left(2 \nabla x_{j}^{1 / 2}+\left(z_{j} x_{j}^{1 / 2}-(x \cdot z) \rho_{j} x_{j}^{-1 / 2}\right) \nabla \Phi\right) .
\end{aligned}
$$

In view of $\sum_{i=1}^{n}(B \nabla w)_{i}=0$, it follows that

$$
\begin{aligned}
& \sum_{i, j=1}^{n}\left(M_{i}^{-1 / 2} x_{i}^{-1 / 2}(z \cdot x) \rho_{i} \nabla \Phi\right)\left(\widetilde{A}_{S}\right)_{i j}^{-1} M_{j}^{-1 / 2} \\
& \times\left(2 \nabla x_{j}^{1 / 2}+\left(z_{j} x_{j}^{1 / 2}-(x \cdot z) \rho_{j} x_{j}^{-1 / 2}\right) \nabla \Phi\right) \\
= & \sum_{i, j=1}^{n}(c(z \cdot x) \nabla \Phi) \tilde{A}_{i j}^{-1}\left(\nabla x_{j}+\left(z_{j} x_{j}-(x \cdot z) \rho_{j} \nabla \Phi\right)\right. \\
= & (c(z \cdot x) \nabla \Phi) \cdot \sum_{i=1}^{n}(B \nabla w)_{i}=0 .
\end{aligned}
$$


Adding this expression to (34), we find that

$$
\begin{aligned}
\nabla w: B \nabla w= & \sum_{i, j=1}^{n} M_{i}^{-1 / 2}\left(2 \nabla x_{i}^{1 / 2}+\left(z_{i} x_{i}^{1 / 2}-(z \cdot x) \rho_{i} x_{i}^{-1 / 2} \nabla \Phi\right)\left(\widetilde{A}_{S}\right)_{i j}^{-1} M_{j}^{-1 / 2}\right. \\
& \times\left(2 \nabla x_{j}^{1 / 2}+\left(z_{j} x_{j}^{1 / 2}-(z \cdot x) \rho_{j} x_{j}^{-1 / 2} \nabla \Phi\right) .\right.
\end{aligned}
$$

The matrix $\widetilde{A}_{S}^{-1}$ is positive definite on $\operatorname{im}\left(\widetilde{A}_{S}\right)=\operatorname{span}\left\{\rho^{1 / 2}\right\}^{\perp}$. A simple computation shows that the vector $M^{-1 / 2}\left(2 \nabla x_{i}^{1 / 2}+\left(z_{i} x_{i}^{1 / 2}-(x \cdot z) \rho_{i} x_{i}^{-1 / 2} \nabla \Phi\right)_{i=1}^{n}\right.$ lies in $\operatorname{span}\left\{\rho^{1 / 2}\right\}^{\perp}$. We obtain

$$
\begin{aligned}
\nabla w: B \nabla w & \geq K_{B} \sum_{i=1}^{n} M_{i}^{-1} \mid 2 \nabla x_{i}^{1 / 2}+\left(z_{i} x_{i}^{1 / 2}-\left.(x \cdot z) \rho_{i} x_{i}^{-1 / 2} \nabla \Phi\right|^{2}\right. \\
& \geq K_{1} \sum_{i=1}^{n}\left|\nabla x_{i}^{1 / 2}\right|^{2}-K_{2} \sum_{i=1}^{n} \mid\left(z_{i} x_{i}^{1 / 2}-\left.(x \cdot z) \rho_{i} x_{i}^{-1 / 2} \nabla \Phi\right|^{2},\right.
\end{aligned}
$$

where $K_{1}>0$ and $K_{2}>0$ depend on $M_{1}, \ldots, M_{n}$. Since $x_{i}$ and $\rho_{i} x_{i}^{-1 / 2}=$ $\left(\rho_{i} c_{\text {tot }} M_{i}\right)^{1 / 2}$ are bounded, the previous inequality becomes

$$
\nabla w: B \nabla w \geq K_{1} \sum_{i=1}^{n}\left|\nabla x_{i}^{1 / 2}\right|^{2}-K_{3}|\nabla \Phi|^{2},
$$

where $K_{3}$ depends on $K_{2}$ and $z_{i}$.

In the following, let $K>0$ be a generic constant independent of $\varepsilon, n$, and $\tau$. We estimate the expression involving the boundary term

$$
\begin{aligned}
\nabla w_{D}: B \nabla w & =\nabla w_{D}: A_{0}^{-1} D^{\prime} \\
& =\sum_{i, j=1}^{n-1}\left(A_{0}^{-1}\right)_{i j}\left(\frac{z_{i}}{M_{i}}-\frac{z_{n}}{M_{n}}\right) \nabla \Phi_{D} \cdot\left(\nabla x_{i}+\left(z_{i} x_{i}-(z \cdot x) \rho_{i}\right) \nabla \Phi\right) \\
& \leq \frac{K}{\delta}+\delta \sum_{i=1}^{n-1}\left|\nabla x_{i}+\left(z_{i} x_{i}-(z \cdot x) \rho_{i}\right) \nabla \Phi\right|^{2},
\end{aligned}
$$

where $K>0$ depends on $\nabla \Phi_{D}, z_{i}, M_{i}$, and $A_{0}^{-1}$. Since $0 \leq x_{i} \leq 1$, we have $\left|\nabla x_{i}\right|^{2}=4 x_{i}\left|\nabla x_{i}^{1 / 2}\right|^{2} \leq 4\left|\nabla x_{i}^{1 / 2}\right|^{2}$ and therefore,

$$
\nabla w_{D}: B \nabla w \leq \frac{K}{\delta}+4 \delta\left|\nabla x_{i}^{1 / 2}\right|^{2}+\delta K|\nabla \Phi|^{2} .
$$

We infer from (35) and (36) that

$$
\int_{\Omega} \nabla\left(w-w_{D}\right): B \nabla w d y \geq\left(K_{1}-4 \delta\right) \sum_{i=1}^{n}\left\|\nabla x_{i}^{1 / 2}\right\|_{L^{2}(\Omega)}^{2}-K_{3}\|\nabla \Phi\|_{L^{2}(\Omega)}^{2}-\frac{K}{\delta} .
$$

By the boundedness of $c_{i}$, the elliptic estimate for the Poisson equation gives

$$
\|\Phi\|_{H^{1}(\Omega)} \leq K\left(1+\left\|c_{i}\right\|_{L^{2}(\Omega)}\right) \leq K .
$$

This proves the lemma. 
Combining the discrete entropy inequality (15) and the estimate of Lemma 8 and summation over $k$ leads to the following result.

Corollary 9 There exist constants $K_{1}>0$ and $K_{2}>0$, both independent of $\varepsilon, n$, and $\tau$, such that

$$
H\left(\rho^{k}\right)+\tau K_{1} \sum_{j=1}^{k} \sum_{i=1}^{n}\left\|\nabla\left(x_{i}^{k}\right)^{1 / 2}\right\|_{L^{2}(\Omega)}^{2}+\varepsilon \tau \sum_{j=1}^{k}\left\|w^{j}-w_{D}\right\|_{L^{2}(\Omega)}^{2} \leq \tau k K_{2}+H\left(\rho^{0}\right) .
$$

Step 2: limit $\boldsymbol{\varepsilon} \rightarrow \mathbf{0}$ For a fixed time step $k$, let $\left(w^{\varepsilon}, \Phi^{\varepsilon}\right)$ be a solution to (12)(13) with $\rho^{\varepsilon}=\rho\left(w^{\varepsilon}, \Phi^{\varepsilon}\right)$ and $x_{i}^{\varepsilon}=\rho_{i}^{\varepsilon} /\left(c_{\text {tot }}^{\varepsilon} M_{i}\right)$. Estimates (37) and (38) yield the following uniform bounds:

$$
\begin{aligned}
\left\|\rho_{i}^{\varepsilon}\right\|_{L^{\infty}(\Omega)}+\left\|x_{i}^{\varepsilon}\right\|_{L^{\infty}(\Omega)} & \leq 2, \quad i=1, \ldots, n, \\
\left\|x_{i}^{\varepsilon}\right\|_{H^{1}(\Omega)}+\left\|\Phi^{\varepsilon}\right\|_{H^{1}(\Omega)}+\varepsilon^{1 / 2}\left\|w_{i}^{\varepsilon}\right\|_{L^{2}(\Omega)} & \leq K,
\end{aligned}
$$

where $K>0$ is independent of $\varepsilon$ and $N$. The bound for $x_{i}^{\varepsilon}$ in $H^{1}(\Omega)$ is a consequence of the bound for $\left(x_{i}^{\varepsilon}\right)^{1 / 2}$ in $H^{1}(\Omega)$ from (38) and the uniform $L^{\infty}$ bound for $x_{i}^{\varepsilon}$ from (39). We claim that $\left(\rho_{i}^{\varepsilon}\right)$ is bounded in $H^{1}(\Omega)$. Indeed, according to Lemma 6 , it holds that $c_{\text {tot }}^{\varepsilon}=\left(\sum_{j=1}^{n} M_{j} x_{j}^{\varepsilon}\right)^{-1}$, and this expression has the uniform lower bound $\left(\max _{i} M_{i}\right)^{-1}$ and the uniform upper bound $\left(\min _{i} M_{i}\right)^{-1}$. Then, since $\left(x_{i}^{\varepsilon}\right)$ is bounded in $H^{1}(\Omega)$, also $\left(c_{\text {tot }}^{\varepsilon}\right)$ is bounded in $H^{1}(\Omega)$. This implies that $\rho_{i}^{\varepsilon}=c_{\text {tot }}^{\varepsilon} M_{i} x_{i}^{\varepsilon}$ is uniformly bounded in $H^{1}(\Omega)$, proving the claim. Observing that the embedding $H^{1}(\Omega) \hookrightarrow L^{2}(\Omega)$ is compact, there exist subsequences, which are not relabeled, such that as $\varepsilon \rightarrow 0$,

$$
\begin{array}{llll}
x_{i}^{\varepsilon} \rightarrow x_{i}, & \rho_{i}^{\varepsilon} \rightarrow \rho_{i}, & \Phi^{\varepsilon} \rightarrow \Phi & \text { strongly in } L^{2}(\Omega), \\
x_{i}^{\varepsilon} \rightarrow x_{i}, & \rho_{i}^{\varepsilon} \rightarrow \rho_{i}, & \Phi^{\varepsilon} \rightarrow \Phi & \text { weakly in } H^{1}(\Omega), \\
\varepsilon w_{i}^{\varepsilon} \rightarrow 0 & & & \text { strongly in } L^{2}(\Omega) .
\end{array}
$$

In view of the $L^{\infty}$ bounds for $\left(x_{i}^{\varepsilon}\right)$ and $\left(\rho_{i}^{\varepsilon}\right)$, the strong convergences for these (sub-) sequences hold in $L^{p}(\Omega)$ for any $p<\infty$. Consequently, $c_{\text {tot }}^{\varepsilon} \rightarrow c_{\text {tot }}:=\sum_{i=1}^{n} \rho_{i} / M_{i}$ strongly in $L^{2}(\Omega)$, and we can identify $\rho_{i}=c_{\text {tot }} M_{i} x_{i}$ for $i=1, \ldots, n$. Furthermore,

$$
c_{i}^{\varepsilon}=\rho_{i}^{\varepsilon} / M_{i} \rightarrow c_{i}:=\rho_{i} / M_{i} \quad \text { strongly in } L^{2}(\Omega), i=1, \ldots, n .
$$

Recalling definition (2) of $D_{i}$, we have

$$
D_{i}^{\varepsilon}=\nabla x_{i}^{\varepsilon}+\left(z_{i} x_{i}^{\varepsilon}-\left(z \cdot x^{\varepsilon}\right) \rho_{i}^{\varepsilon}\right) \nabla \Phi^{\varepsilon} \rightarrow D_{i}:=\nabla x_{i}+\left(z_{i} x_{i}-(z \cdot x) \rho_{i}\right) \nabla \Phi
$$

weakly in $L^{q}(\Omega)$ for any $q<2$ and $i=1, \ldots, n$. Since $\left(D_{i}^{\varepsilon}\right)$ is bounded in $L^{2}(\Omega)$, there exists a subsequence which converges to some function $\widetilde{D}_{i}$ weakly in $L^{2}(\Omega)$. By the uniqueness of the weak limits, we can identify $\widetilde{D}_{i}=D_{i}$. This shows that the convergence (41) holds in $L^{2}(\Omega)$. We deduce from the strong convergence of $\left(x_{i}^{\varepsilon}\right)$, the boundedness of $\left(x_{i}^{\varepsilon}\right)$ in $L^{\infty}(\Omega)$, and the continuity of $r_{i}$ that $r_{i}\left(x^{\varepsilon}\right) \rightarrow r_{i}(x)$ strongly in $L^{2}(\Omega)$.

We know from Lemma 4 that $B\left(w^{\varepsilon}\right) \nabla w^{\varepsilon}=A_{0}^{-1}\left(\rho^{\varepsilon}\right)\left(D^{\varepsilon}\right)^{\prime}$. As $A_{0}^{-1}(\rho)$ is uniformly bounded for $\rho \in[0,1]^{n}$ and $\left(\rho^{\varepsilon}\right)$ converges strongly to $\rho$, we infer that 
$A_{0}^{-1}\left(\rho^{\varepsilon}\right) \rightarrow A_{0}^{-1}(\rho)$ strongly in $L^{2}(\Omega)$; the convergence holds even in every $L^{p}(\Omega)$ for $p<\infty$. Then, because of (41) and using the continuity of $\rho \mapsto A_{0}^{-1}(\rho)$,

$$
A_{0}^{-1}\left(\rho^{\varepsilon}\right)\left(D^{\varepsilon}\right)^{\prime} \rightarrow A_{0}^{-1}(\rho) D^{\prime} \quad \text { weakly in } L^{q}(\Omega) \text { for all } q<2 \text {. }
$$

In fact, since $\left.A_{0}^{-1}\left(\rho^{\varepsilon}\right)\left(D^{\varepsilon}\right)^{\prime}\right)$ is bounded in $L^{2}(\Omega)$ and thus (up to a subsequence) weakly converging in $L^{2}(\Omega)$, the convergence holds in $L^{2}(\Omega)$.

These convergences are sufficient to perform the limit $\varepsilon \rightarrow 0$ in (12)-(13). We conclude that $\left(\rho^{k}, \Phi^{k}\right):=(\rho, \Phi)$ solves

$$
\begin{aligned}
& \frac{1}{\tau} \int_{\Omega}\left(\left(\rho^{k}\right)^{\prime}-\left(\rho^{k-1}\right)^{\prime}\right) \cdot \phi d y+\int_{\Omega} \nabla \phi: A_{0}^{-1}\left(\rho^{k}\right) \nabla \rho^{k} d y=\int_{\Omega} r^{\prime}\left(x^{k}\right) \cdot \phi d y(43) \\
& \lambda \int_{\Omega} \nabla \Phi^{k} \cdot \nabla \theta d y=\int_{\Omega}\left(\sum_{i=1}^{n} z_{i} c_{i}^{k}+f(y)\right) \theta d y
\end{aligned}
$$

for all $\phi \in V_{N}, \theta \in P_{N}$.

Step 3: limit $N \rightarrow \infty$ Let $\left(\rho^{N}, \Phi^{N}\right)$ be a solution to (43)-(44). Estimates (39)-(40) are independent of $N$. Thus, we can exactly argue as in step 2 and obtain limit functions $(x, \rho, \Phi)$ and $c_{i}=c_{\text {tot }} M_{i} x_{i}$ for $i=1, \ldots, n$ as $N \rightarrow \infty$. These functions satisfy (43)-(44) for all $\phi \in V_{N}$ and $\theta \in P_{N}$ and for all $N \in \mathbb{N}$. The union of all $V_{N}$ is dense in $H^{1}\left(\Omega ; \mathbb{R}^{n-1}\right)$ and the union of all $P_{N}$ is dense in $H_{D}^{1}(\Omega)$. Thus, by a density argument, system (43)-(44) hold for all test functions $\phi \in H^{1}\left(\Omega ; \mathbb{R}^{n-1}\right)$ and $\theta \in H_{D}^{1}(\Omega)$.

Step 4: limit $\tau \rightarrow 0$. Let $\left(\rho^{k}, \Phi^{k}\right)$ be a solution to (43)-(44) with test functions $\phi \in H^{1}\left(\Omega ; \mathbb{R}^{n-1}\right)$ and $\theta \in H_{D}^{1}(\Omega)$. Then, $\rho_{i}^{k}=c_{\text {tot }}^{k} M_{i} x_{i}^{k}$ and $c_{i}^{k}=\rho_{i}^{k} / M_{i}$ for $i=1, \ldots, n$. We set

$$
\rho_{i}^{\tau}(y, t)=\rho_{i}^{k}(y), \quad x_{i}^{\tau}(y, t)=x_{i}^{k}(y), \quad c_{i}^{\tau}(y, t)=c_{i}^{k}(y), \quad \Phi^{\tau}(y, t)=\Phi^{k}(y)
$$

for $y \in \Omega, t \in((k-1) \tau, k \tau], i=1, \ldots, n$ and introduce the shift operator $\left(\sigma_{\tau} \rho^{\tau}\right)(y, t)=\rho^{k-1}(y)$ for $y \in \Omega$ and $t \in((k-1) \tau, k \tau]$. Finally, we set $D_{i}^{\tau}=\nabla x_{i}^{\tau}+\left(z_{i} x_{i}^{\tau}-\left(z \cdot x^{\tau}\right) \rho_{i}^{\tau}\right) \nabla \Phi^{\tau}$ and $T=m \tau$ for some fixed $m \in \mathbb{N}$. Then, we can write system (43)-(44) as

$$
\begin{aligned}
& \frac{1}{\tau} \int_{0}^{T} \int_{\Omega}\left(\left(\rho^{\tau}\right)^{\prime}-\sigma_{\tau}\left(\rho^{\tau}\right)^{\prime}\right) \cdot \phi d y d t+\int_{0}^{T} \int_{\Omega} \nabla \phi: A_{0}^{-1}\left(\rho^{\tau}\right)\left(D^{\tau}\right)^{\prime} d y d t \\
= & \int_{0}^{t} \int_{\Omega} r^{\prime}\left(x^{\tau}\right) \cdot \phi d y d t \\
& \lambda \int_{\Omega} \nabla \Phi^{\tau} \cdot \nabla \theta d y=\int_{\Omega}\left(\sum_{i=1}^{n} z_{i} c_{i}^{\tau}+f(y)\right) \theta d y
\end{aligned}
$$

for all piecewise constant functions $\phi:(0, T) \rightarrow H^{1}\left(\Omega ; R^{n-1}\right)$ and $\theta:(0, T) \rightarrow$ $H_{D}^{1}(\Omega)$. The entropy inequality (38), formulated in terms of $\left(\rho^{\tau}, \Phi^{\tau}\right)$, provides us with further uniform bounds since the right-hand side of (38) does not depend on $\tau$ :

$$
\begin{aligned}
\left\|\rho_{i}^{\tau}\right\|_{L^{\infty}\left(\Omega_{T}\right)}+\left\|x_{i}^{\tau}\right\|_{L^{\infty}\left(\Omega_{T}\right)} & \leq K, \\
\left\|\rho_{i}^{\tau}\right\|_{L^{2}\left(0, T ; H^{1}(\Omega)\right)}+\left\|x_{i}^{\tau}\right\|_{L^{2}\left(0, T ; H^{1}(\Omega)\right)}+\left\|\Phi^{\tau}\right\|_{L^{2}\left(0, T ; H^{1}(\Omega)\right)} & \leq K,
\end{aligned}
$$


where we have set $\Omega_{T}=\Omega \times(0, T)$. As a consequence, $\left(D_{i}^{\tau}\right)$ is bounded in $L^{2}\left(0, T ; H^{1}(\Omega)\right)$.

It remains to derive a uniform estimate for the discrete time derivative of $\rho^{\tau}$. Taking into account the uniform bound for $A_{0}^{-1}\left(\rho^{\tau}\right)$, it follows that

$$
\begin{aligned}
& \frac{1}{\tau}\left|\int_{0}^{T} \int_{\Omega}\left(\left(\rho^{\tau}\right)^{\prime}-\sigma_{\tau}\left(\rho^{\tau}\right)^{\prime}\right) \cdot \phi d y d t\right| \\
\leq & \int_{0}^{T}\|\nabla \phi\|_{L^{2}(\Omega)}\left\|A_{0}^{-1}\left(\rho^{\tau}\right)\right\|_{L^{\infty}(\Omega)}\left\|\left(D^{\tau}\right)^{\prime}\right\|_{L^{2}(\Omega)} d t \\
& +\int_{0}^{T}\left\|r^{\prime}\left(x^{\tau}\right)\right\|_{L^{2}(\Omega)}\|\phi\|_{L^{2}(\Omega)} d t \leq C\|\phi\|_{L^{2}\left(0, T ; H^{1}(\Omega)\right)} .
\end{aligned}
$$

As the piecewise constant functions $\phi:(0, T) \rightarrow H^{1}\left(\Omega ; \mathbb{R}^{n-1}\right)$ are dense in $L^{2}\left(0, T ; H^{1}\left(\Omega ; \mathbb{R}^{n-1}\right)\right)$, this estimate also holds for all $\phi \in L^{2}\left(0, T ; H^{1}\left(\Omega ; \mathbb{R}^{n-1}\right)\right)$, and we conclude that

$$
\tau^{-1}\left\|\left(\rho^{\tau}\right)^{\prime}-\sigma_{\tau}\left(\rho^{\tau}\right)^{\prime}\right\|_{L^{2}\left(0, T ; H^{1}(\Omega)^{\prime}\right)} \leq K, \quad i=1, \ldots, n-1 .
$$

This estimate also holds for $i=n$ since $\rho_{n}^{\tau}=1-\sum_{i=1}^{n-1} \rho_{i}^{\tau}$.

By the Aubin-Lions lemma in the version of [12], there exists a subsequence of $\left(\rho^{\tau}\right)$ which is not relabeled such that, as $\tau \rightarrow 0$,

$$
\rho_{i}^{\tau} \rightarrow \rho_{i} \quad \text { strongly in } L^{2}\left(\Omega_{T}\right), i=1, \ldots, n .
$$

In view of the $L^{\infty}$ bound (47) for $\rho^{\tau}$, this convergence also holds in $L^{p}\left(\Omega_{T}\right)$ for any $p<\infty$. Furthermore, by (48), we have up to subsequences,

$$
\begin{array}{ll}
\stackrel{\tau}{i} x_{i}, \quad \Phi^{\tau} \rightarrow \Phi & \text { weakly in } L^{2}\left(0, T ; H^{1}(\Omega)\right), \\
\tau^{-1}\left(\rho_{i}^{\tau}-\sigma_{\tau}\left(\rho_{i}^{\tau}\right)\right) \rightarrow \partial_{t} \rho_{i} & \text { weakly in } L^{2}\left(0, T ; H^{1}(\Omega)^{\prime}\right) .
\end{array}
$$

In particular, $D_{i}^{\tau} \rightarrow D_{i}$ weakly in $L^{2}\left(\Omega_{T}\right)$, and we can identify $D_{i}=\nabla x_{i}+\left(z_{i} x_{i}-\right.$ $\left.(z \cdot x) \rho_{i}\right) \nabla \Phi$. The strong convergence of $\left(\rho^{\tau}\right)$ and the weak convergence of $\left(D_{i}^{\tau}\right)$ imply that

$$
A_{0}^{-1}\left(\rho^{\tau}\right)\left(D^{\tau}\right)^{\prime} \rightarrow A_{0}^{-1}(\rho) D^{\prime} \quad \text { weakly in } L^{q}\left(\Omega_{T}\right), q<2 .
$$

Again, since $\left(A_{0}^{-1}\left(\rho^{\tau}\right)\left(D^{\tau}\right)^{\prime}\right)$ is bounded in $L^{2}\left(\Omega_{T}\right)$, this convergence holds in $L^{2}\left(\Omega_{T}\right)$. Furthermore, $r^{\prime}\left(x^{\tau}\right) \rightarrow r^{\prime}(x)$ strongly in $L^{2}\left(\Omega_{T}\right)$. Therefore, we can pass to the limit $\tau \rightarrow 0$ in (45)-(46) yielding (16)-(17).

\section{Numerical experiments}

In this section, some numerical experiments based on scheme (12)-(13) in one space dimension are presented. We stress the fact that the experiments just serve as a feasibility study and more effort is necessary to perform two- or three-dimensional simulations showing, for instance, the effects coming from the mixed boundary 
conditions. In the context of semiconductor simulations, we refer to [16]. The onedimensional setting presented here models liquid electrolytes, which can be used as a simplified version of a model for dye-sensitized solar cells [35].

\subsection{Discretization and iteration procedure}

Let $\Omega=(0,1)$ be divided into $n_{p} \in \mathbb{N}$ uniform subintervals of length $h=1 / n_{p}$. We use uniform time steps with time step size $\tau>0$ and piecewise linear finite elements with the usual conforming P1 finite-element space on the uniform partition, i.e.,

$$
\begin{aligned}
& P_{N}:=\left\{p \in C(\bar{\Omega}):\left.p\right|_{I} \text { is affine on each subinterval } I\right\}, \\
& V_{N}:=\left\{v \in C\left(\bar{\Omega} ; \mathbb{R}^{n-1}\right):\left.v\right|_{I} \text { is affine on each subinterval } I\right\} .
\end{aligned}
$$

We impose Dirichlet boundary condition for the electric potential $\Phi: \Phi(0)=0$ and $\Phi(1)=U$, where $U$ is the applied voltage. Given the variables $(w, \Phi)$, the molar fractions $x_{i}$ are computed from the fixed-point problem (see the proof of Lemma 5)

$$
f(s)=\sum_{i=1}^{n-1}(1-s)^{M_{i} / M_{n}} \exp \left[M_{i} w_{i}-M_{i}\left(\frac{z_{i}}{M_{i}}-\frac{z_{n}}{M_{n}}\right) \Phi_{0}\right], \quad s \in[0,1],
$$

with unique solution $s_{0} \in(0,1)$. This nonlinear set of equations is solved by the MATLAB routine fzero. The molar fractions are recovered from (28),

$$
x_{i}=\left(1-s_{0}\right)^{M_{i} / M_{n}} \exp \left[M_{i} w_{i}-M_{i}\left(\frac{z_{i}}{M_{i}}-\frac{z_{n}}{M_{n}}\right) \Phi\right], \quad i=1, \ldots, n-1,
$$

and $x_{n}=1-s_{0}$. Then, we set (see Lemma 6) $c_{\text {tot }}=\left(\sum_{i=1}^{n} M_{i} x_{i}\right)^{-1}$ and $\rho_{i}=c_{\text {tot }} M_{i} x_{i}$ for $i=1, \ldots, n$. Note that we can compute the diffusion matrix $B(\rho(w, \Phi))$, given $w$ and $\Phi$, explicitly from the formula $B(\rho(w, \Phi))=$ $c_{\text {tot }} C^{-1}(\rho(w, \Phi))$, where the matrix $C(\rho)$ is defined in Section 3.1.

Instead of solving the nonlinear discrete system (12)-(13) by a full Newton method, we employ a linearized semi-implicit approach, i.e., we linearize $\rho(w, \Phi)$ and use the previous time step in the diffusion matrix $B(w)$. More precisely, let $\bar{w} \in V_{N}$ and $\bar{\Phi} \in P_{N}$ be given. We linearize $\rho(w, \Phi)$ by

$$
\rho(\bar{w}, \bar{\Phi})+\nabla_{(w, \Phi)} \rho^{\prime}(\bar{w}, \bar{\Phi}) \cdot(w-\bar{w}, \Phi-\bar{\Phi}) .
$$

This leads to the problem in the variable $\zeta=(w-\bar{w}, \Phi-\bar{\Phi})$ :

$$
L(\zeta, \phi)=F(\phi), \quad K\left(\zeta_{n}, \theta\right)=G(\theta) \quad \text { for all } \phi \in V_{N}, \theta \in P_{N},
$$

where

$$
\begin{aligned}
L(\zeta, \phi)= & \int_{\Omega} \nabla_{(w, \Phi)} \rho^{\prime}(\bar{w}, \bar{\Phi}) \cdot(\zeta, \phi) d y+\tau \int_{\Omega} \partial_{x} \phi \cdot B(\bar{w}, \bar{\Phi}) \partial_{x} \zeta d y \\
& +\varepsilon \tau \int_{\Omega}\left(\zeta-w_{D}\right) \cdot \phi d y \\
F(\phi)= & -\int_{\Omega}\left(\rho^{\prime}(\bar{w}, \bar{\Phi})-\rho^{\prime}\left(w^{k-1}, \Phi^{k-1}\right)\right) \cdot \phi d y-\tau \int_{\Omega} \partial_{x} \phi \cdot B(\bar{w}, \bar{\Phi}) \partial_{x} \bar{w} d y,
\end{aligned}
$$




$$
\begin{aligned}
K\left(\zeta_{n}, \theta\right) & =\lambda \int_{\Omega} \partial_{x} \zeta_{n} \partial_{x} \phi d y-\int_{\Omega} \sum_{i=1}^{n} \frac{z_{i}}{M_{i}} \nabla_{(w, \Phi)} \rho_{i}(\bar{w}, \bar{\Phi}) \cdot \zeta \theta d y, \\
G(\theta) & =-\lambda \int_{\Omega} \partial_{x} \bar{\Phi} \partial_{x} \theta d y+\int_{\Omega}\left(\sum_{i=1}^{n} z_{i} \frac{\rho_{i}(\bar{w}, \bar{\Phi})}{M_{i}}+f(y)\right) \theta d y .
\end{aligned}
$$

The iteration with starting point $\left(w_{h}^{(0)}, \Phi_{h}^{(0)}\right):=\left(w^{k-1}, \Phi^{k-1}\right)$ is then defined by $\left(w_{h}^{(m+1)}, \Phi_{h}^{(m+1)}\right):=(\bar{w}, \bar{\Phi})+\zeta$ for $m \geq 0$. The iteration stops when $\|\zeta\|_{\ell^{\infty}}<\varepsilon_{\text {tol }}$ for some tolerance $\varepsilon_{\text {tol }}>0$ or if $m \geq m_{\max }$ for a maximal number of iterations. We summarize the scheme in Algorithm 1.

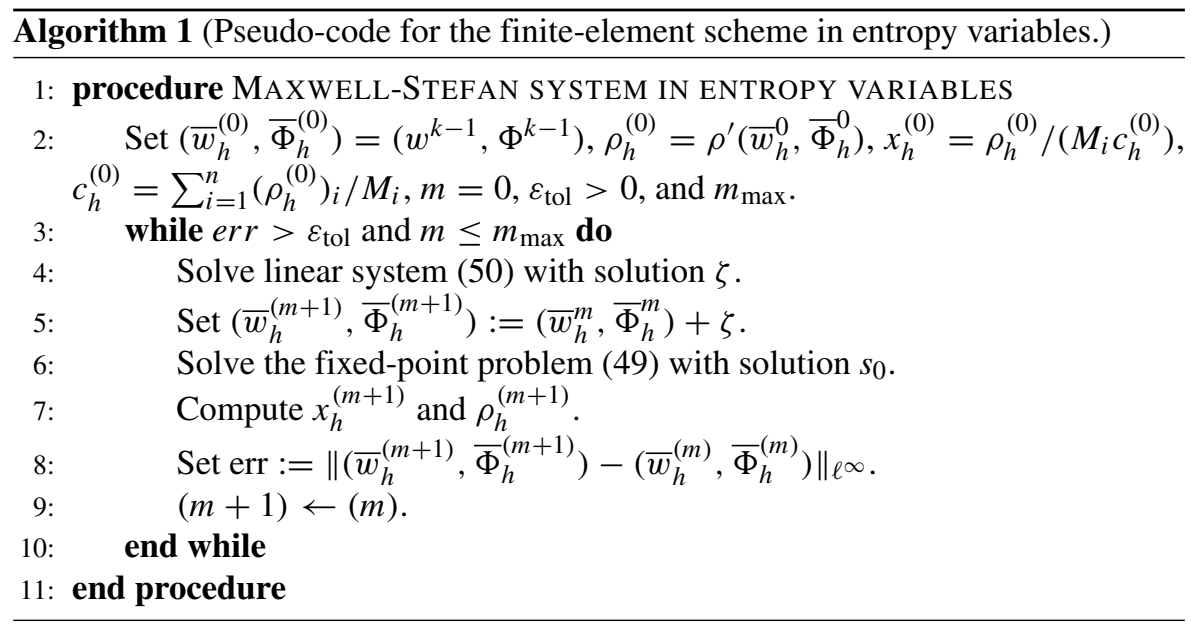

All integrals appearing in the scheme are computed by a three-point Gaussian quadrature rule. The matrix $B=c_{\text {tot }} C^{-1}$ is evaluated explicitly, where $C$ is the matrix from Section 3.1. The linear system (50) and the fixed-point problem (49) are solved using MATLAB. We choose the numerical parameters $h=10^{-2}, \tau=10^{-3}$, $\varepsilon_{\text {tol }}=10^{-12}$, and $\varepsilon=2^{-52} \approx 2.2204 \cdot 10^{-16}$ (the scheme works also for $\varepsilon=0$ ). The linearization procedure in this implementation is not captured by our analysis, since this would be beyond the scope of this paper and is postponed to future work. A possible approach in this direction could be a combination of the scalar results in [7] to ensure stability of the linearized system and [5] to show the convergence of the linearization procedure. We have compared our results with the solutions from a finite-element scheme derived from the original system in the variables $\rho_{i}$ and a Picard iteration procedure for the nonlinear discrete system. It turned out that the results are basically the same, i.e., $\left\|\rho_{i}-\rho_{i}(w, \Phi)\right\|_{L^{\infty}(\Omega)} \leq 10^{-10}$.

\subsection{Numerical examples}

In all numerical examples, we neglect reaction terms, set $m_{\max }=200$, and choose the diffusivities according to $[3,17]: D_{12}=0.833, D_{13}=0.680$, and $D_{23}=0.168$ 
for $n=3$. The charges are given by $z_{1}=z_{2}=1$ and $z_{3}=0$ and the initial data is defined as in [3]:

$$
\rho_{1}^{0}(y)= \begin{cases}0.7 & \text { for } y<0.25 \\ -2(0.7-\eta) y-2(0.25 \eta-(0.7 \cdot 0.75)) & \text { for } 0.25 \leq y<0.75 \\ \eta & \text { for } 0.75 \leq y \leq 1\end{cases}
$$

for $\eta=2^{-52}, \rho_{2}^{0}(y)=0.2$, and $\rho_{3}^{0}(y)=\left(1-\rho_{1}^{0}-\rho_{2}^{0}\right)(y)$ for $y \in \Omega=(0,1)$. The parameter $\eta>0$ is needed to transform the initial conditions to the entropy variables. It can be avoided by changing the first step of the iteration procedure but since $\eta$ equals the machine precision, there is practically no difference in the results.

For the first example, we suppose that all three molar masses are equal to one and that the boundary conditions for the electric potential are in equilibrium, i.e., $M_{1}=M_{2}=M_{3}=1$ and $\Phi(y)=0$ for $y \in\{0,1\}$. The dynamics of the particle densities and the electric potential are shown in Fig. 1. The solution at time $t=17$ is essentially stationary and, in fact, in equilibrium. Because of the choice of the parameters, the stationary solution is symmetric around $x=\frac{1}{2}$.

In order to study the convergence of our iterative linearization procedure, we plot the evolution of the iteration parameter $m$ over time in Fig. 2. It turns out that the
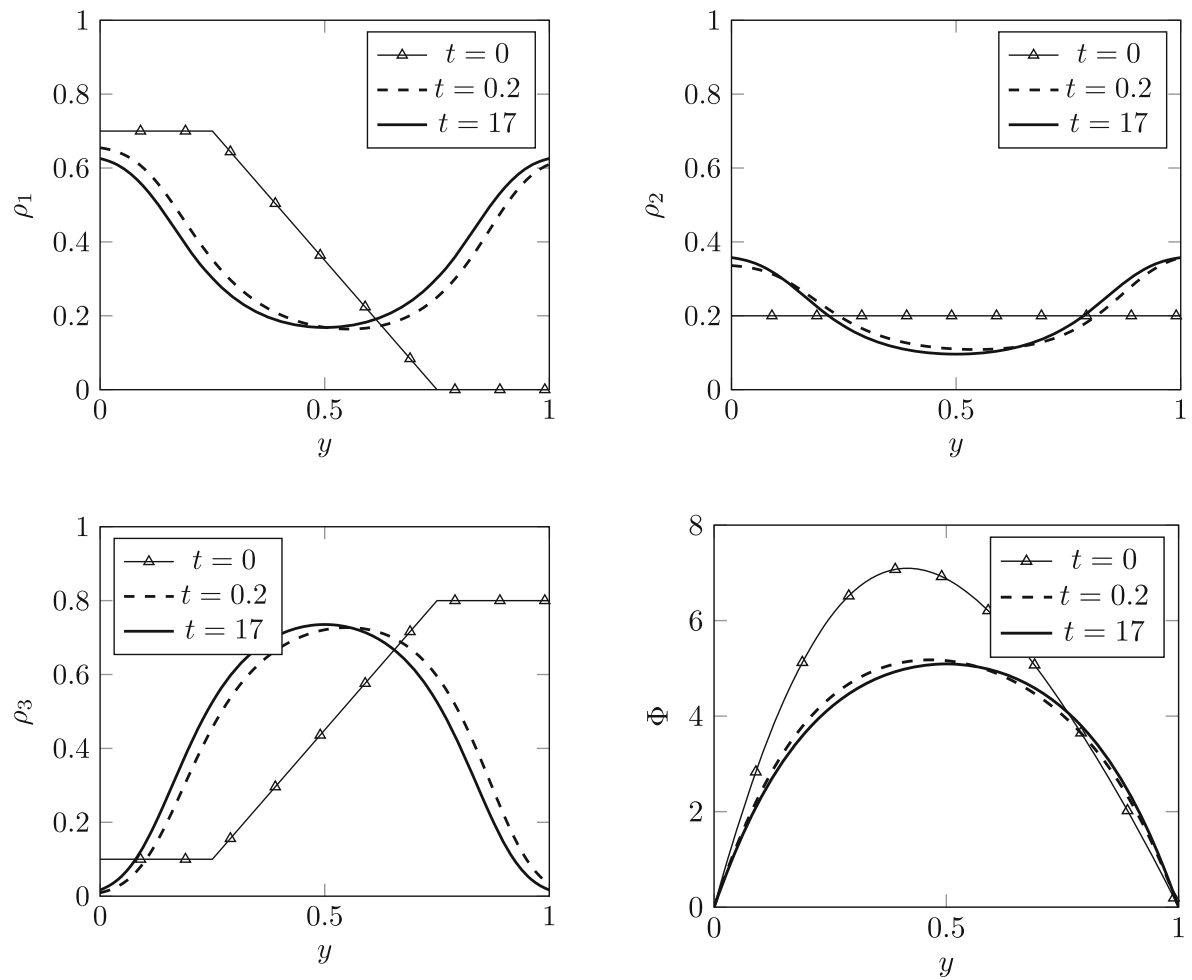

Fig. 1 Example 1: Particle densities $\rho_{i}$ and electric potential for molar masses $M_{1}=M_{2}=M_{3}=1$ versus position at various times. The boundary conditions for the electric potential are in equilibrium 


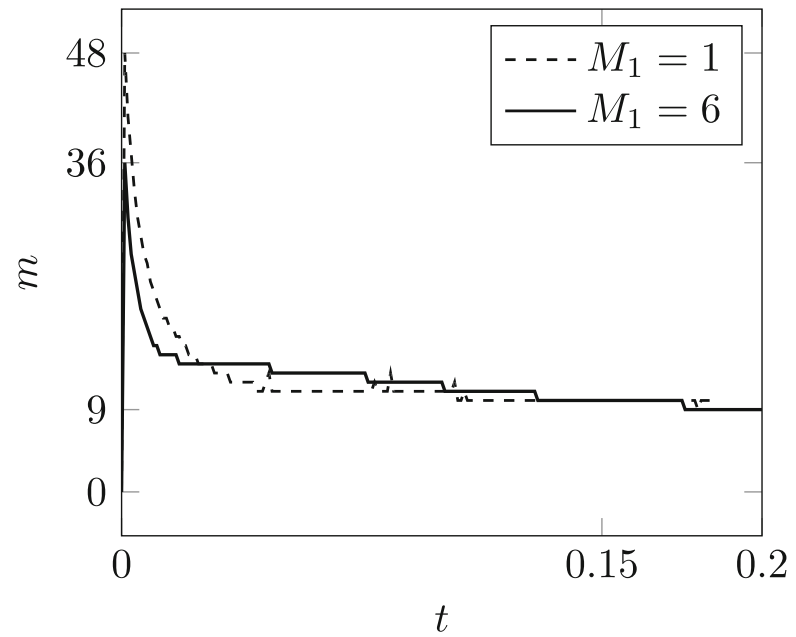

Fig. 2 Examples 1 and 2: Plot of the iteration parameter $m$ versus time $t$. After $t \geq 0.2$, iterations stop at $m=9$ in both examples
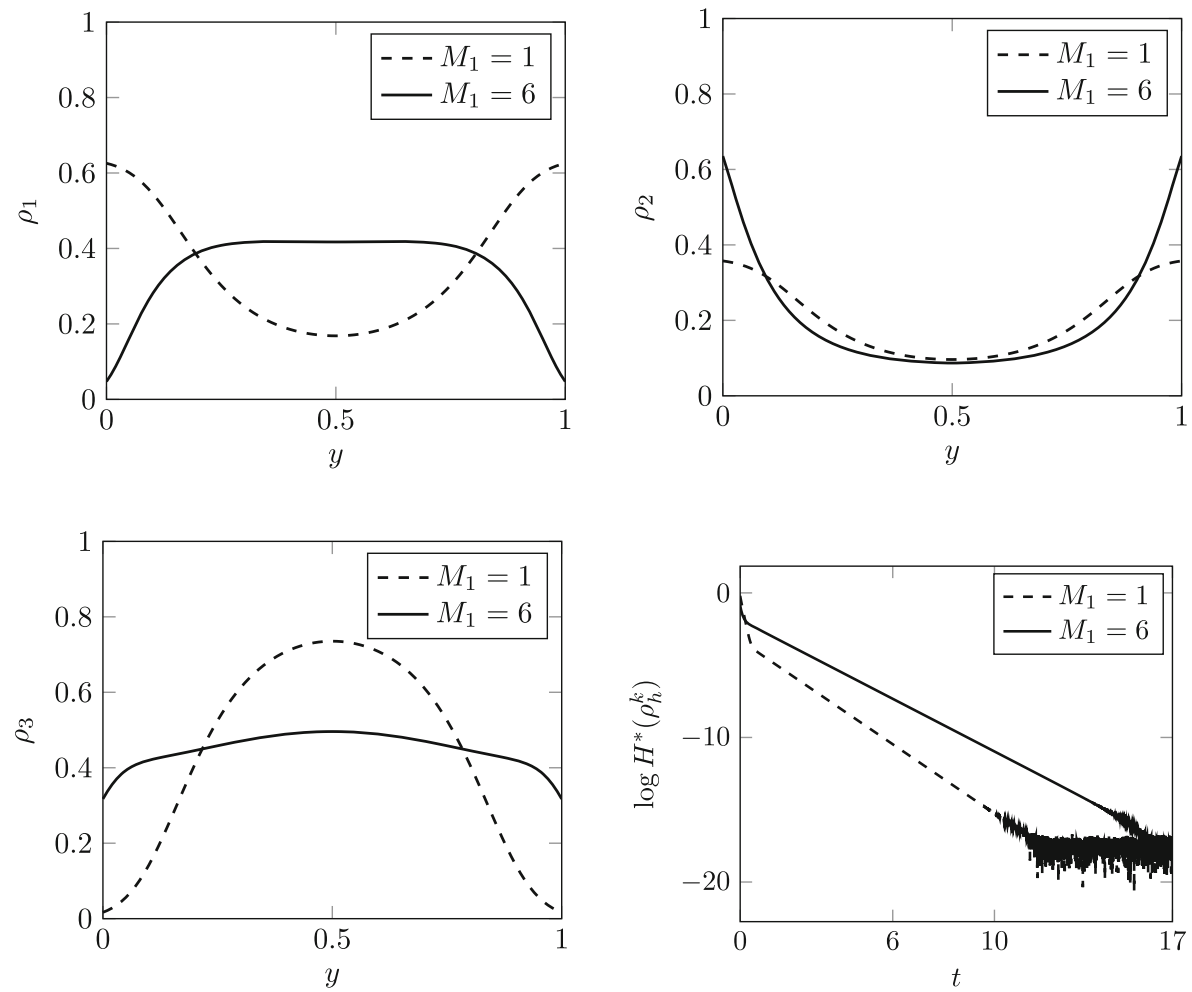

Fig. 3 Example 2: Particle densities $\rho_{i}$ at time $t=4$ versus position and relative entropy (bottom right) for molar masses $M_{1}=6$ and $M_{2}=M_{3}=1$. The boundary conditions for the electric potential are in equilibrium 
number of iterations is decreasing as time progresses. Initially, the algorithm needs $m=48$ iterations and for $t \geq 0.2$ only $m=9$ iterations. The maximum number $m_{\max }=200$ was never reached in all examples and the maximum number of iteration was always beyond $m=50$.

The situation changes drastically when the molar masses are different (example 2). Figure 3 shows the stationary solutions with the same parameters as in the previous example except $M_{1}=6$. Here, the discrete relative entropy is defined by

$$
H^{*}\left(\rho_{h}^{k}\right)=\int_{0}^{1}\left(c_{\text {tot }, h}^{k} \sum_{i=1}^{n}\left(x_{h}^{k}\right)_{i} \log \frac{\left(x_{h}^{k}\right)_{i}}{\left(x_{h}^{\infty}\right)_{i}}+\frac{\lambda}{2}\left|\nabla\left(\Phi_{h}^{k}-\Phi_{h}^{\infty}\right)\right|^{2}\right) d y,
$$

where $\left(\rho_{h}^{k}, \Phi_{h}^{k}\right)$ is the finite-element solution at time $k \tau$ and $\left(x_{h}^{\infty}, \Phi_{h}^{\infty}\right)$ is the stationary solution. The integral and gradients are computed by the trapezoidal and gradient routines of MATLAB. The semi-logarithmic plot of the relative entropy shows that the entropy converges to zero exponentially fast.

For example 3, we choose the same initial conditions and parameters as before, but we take nonequilibrium boundary data $\Phi(0)=10, \Phi(1)=0$. The solutions at time $t=8$ for various molar masses $M_{1}$ are displayed in Fig. 4. Since $\rho_{1}$ and $\rho_{2}$ have
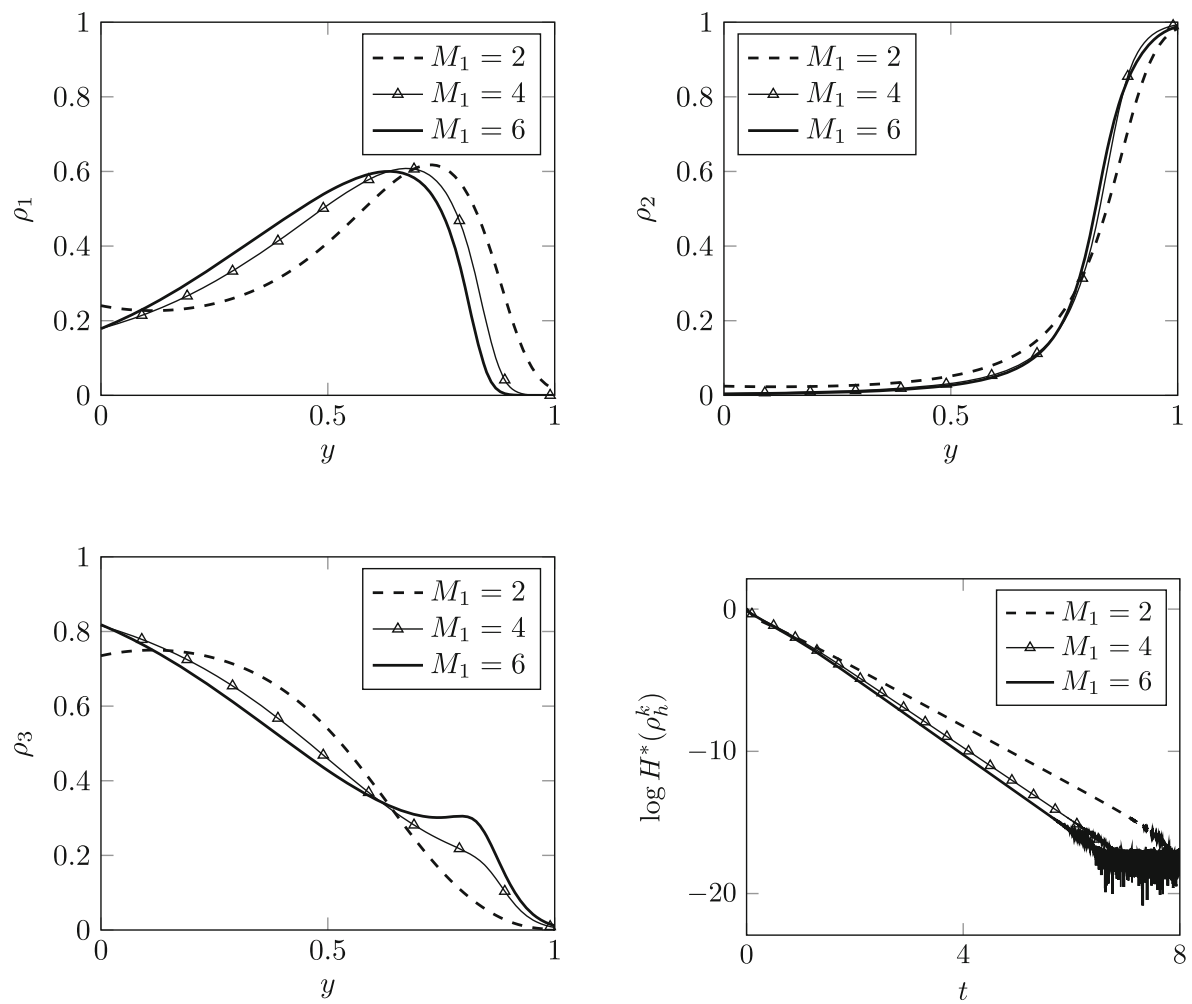

Fig. 4 Example 3: Particle densities $\rho_{i}$ at time $t=8$ versus position and relative entropy (bottom right) for various molar masses $M_{1}$. The boundary conditions for the electric potential are not in equilibrium 

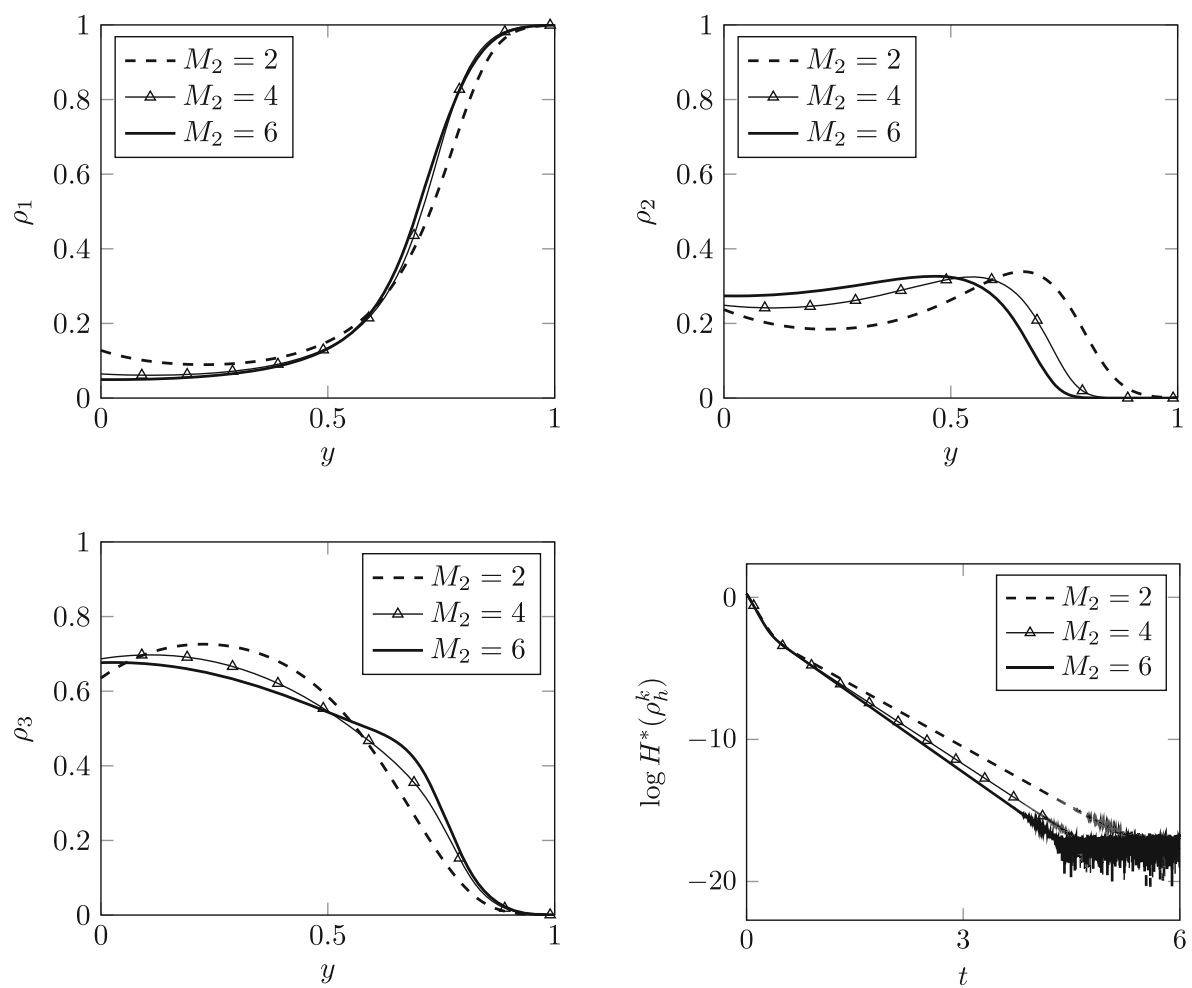

Fig. 5 Example 4: Particle densities $\rho_{i}$ at time $t=8$ versus position and relative entropy (bottom right) for various molar masses $M_{2}$. The boundary conditions for the electric potential are not in equilibrium

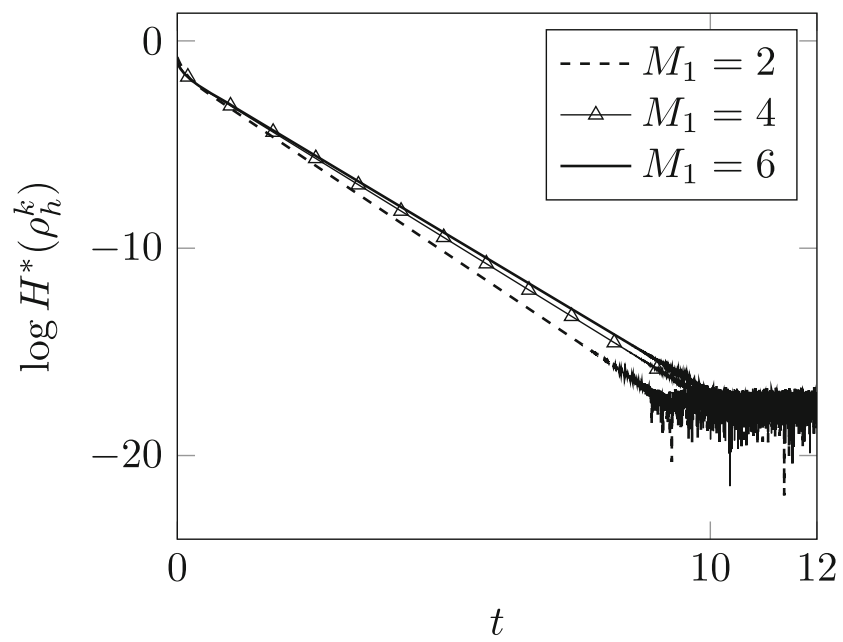

Fig. 6 Example 5: Semi-logarithmic plot of the relative entropy $H^{*}\left(\rho_{h}^{k}\right)$ versus time, without electric potential and for different molar masses 
both positive charge and the potential on the left boundary is positive, both species avoid the left boundary and move to the right.

In example 4 , we interchange the roles of $M_{1}$ and $M_{2}$, i.e., we choose $M_{1}=1$ and $M_{2} \in\{2,4,6\}$. We observe in Fig. 5 that the first species is more concentrated at the right boundary while in the previous example, this holds true for the second species.

The previous examples show that the convergence rate to equilibrium strongly depends on the ratio of the molar masses. It turns out that this effect is triggered by the drift term, and without electric field, the convergence rates are similar for different molar masses. This behavior can be observed in Fig. 6 (example 5), where we have taken the same parameters as in the previous example but neglect the electric field. In this situation, the steady state is constant in space and explicitly computable; indeed, we have $\rho_{i}^{\infty}=\operatorname{mean}(\Omega)^{-1}\left\|\rho_{i}^{0}\right\|_{L^{1}(\Omega)}$. Note that the steady state in the previous examples is not constant.

Finally, we compute the numerical convergence rate when the grid size tends to zero for the situation of example 3 (nonequilibrium boundary conditions for the potential). We choose the time $t=0.01$ and the time step size $\tau=10^{-4}$. The solutions are computed on nested meshes with grid sizes $h \in\{0.01,0.005,0.0025$, $0.0006,0.0001\}$ and compared to the reference solution, computed on a very fine mesh with 25601 elements $\left(h \approx 4 \cdot 10^{-5}\right.$ ). As expected, we observe a second-order convergence in space; see Fig. 7.
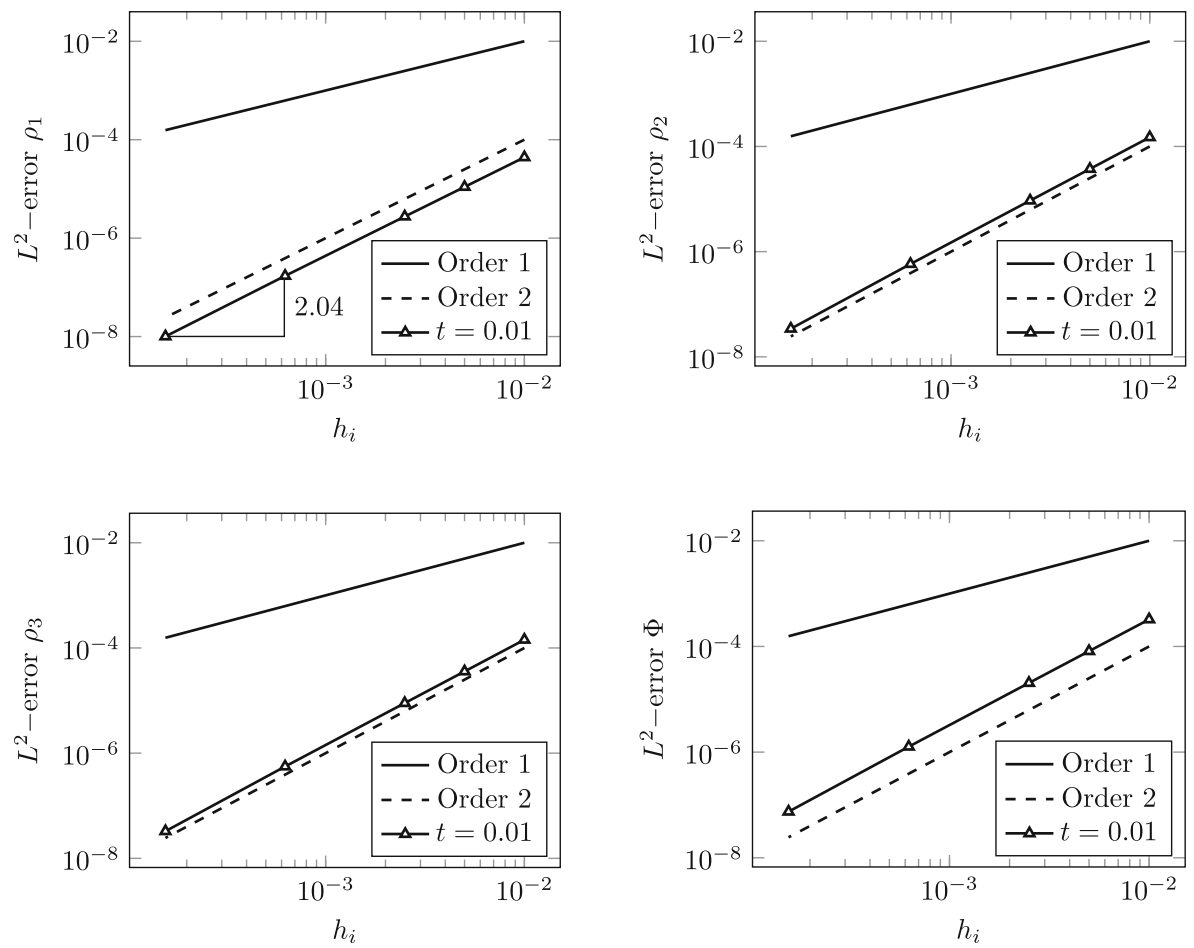

Fig. 7 Discrete $L^{2}$-error relative to the reference solution for the densities and the potential (bottom right) at time $t=0.01$ 
Acknowledgments Open access funding provided by Austrian Science Fund (FWF).

Open Access This article is distributed under the terms of the Creative Commons Attribution 4.0 International License (http://creativecommons.org/licenses/by/4.0/), which permits unrestricted use, distribution, and reproduction in any medium, provided you give appropriate credit to the original author(s) and the source, provide a link to the Creative Commons license, and indicate if changes were made.

Publisher's note Springer Nature remains neutral with regard to jurisdictional claims in published maps and institutional affiliations.

\section{References}

1. Bothe, D. et al.: On the Maxwell-Stefan Equations to Multicomponent Diffusion. In: Escher, J. (ed.) Parabolic Problems. Progress in Nonlinear Differential Equations and Their Applications, pp. 81-93. Springer, Basel (2011)

2. Bothe, D., Dreyer, W.: Continuum thermodynamics of chemically reacting fluid mixtures. Acta Mech. 226, 1757-1805 (2015)

3. Boudin, L., Grec, B., Salvarani, F.: A mathematical and numerical analysis of the Maxwell-Stefan diffusion equations. Discret. Cont. Dyn. Sys. B 17, 1427-1440 (2012)

4. Boudin, L., Grec, B., Pavić, M., Salvarani, F.: Diffusion asymptotics of a kinetic model for gaseous mixtures. Kinetic Related Model 6, 137-157 (2013)

5. Cancès, C., Nabet, F., Vohralik, M.: Convergence and a posteriori error analysis for energy-stable finite element approximations of degenerate parabolic equations. Preprint, 2018. hal-01894884

6. Carnes, B., Carey, G.: Local boundary value problems for the error in FE approximation of non-linear diffusion systems. Intern. J. Numer. Meth Engrg. 73, 665-684 (2008)

7. Chatzipantelidis, P., Horváth, Z., Thomée, V.: On preservation of positivity in some finite element methods for the heat equation. Comput. Meth. Appl. Math. 15, 417-437 (2015)

8. Chen, X., Jüngel, A.: Analysis of an incompressible Navier-Stokes-Maxwell-Stefan system. Commun. Math Phys. 340, 471-497 (2015)

9. Daus, E., Jüngel, A., Tang, B.-Q.: Exponential time decay of solutions to reaction-cross-diffusion systems of Maxwell-Stefan type. Submitted for publication, arXiv:1802.10274 (2018)

10. Desvillettes, L., Fellner, K., Pierre, M., Vovelle, J.: Global existence for quadratic systems of reactiondiffusion. Adv Nonlin. Stud. 7, 491-511 (2007)

11. Dieter-Kisling, K., Marschall, H., Bothe, D.: Numerical method for coupled interfacial surfactant transport on dynamic surface meshes of general topology. Comput. Fluids 109, 168-184 (2015)

12. Dreher, M., Jüngel, A.: Compact families of piecewise constant functions in $L^{p}(0, T ; B)$. Nonlin. Anal. 75, 3072-3077 (2012)

13. Dreyer, W., Druet, P.-E., Gajewski, P., Guhlke, C.: Analysis of improved Nernst-Planck-Poisson models of compressible isothermal electrolytes. Part I Derivation of the model and survey of the results. WIAS Berlin, Germany, preprint no. 2395 (2017)

14. Dreyer, W., Guhlke, C., Müller, R.: Overcoming the shortcomings of the Nernst-Planck-Poisson model. Phys. Chem. Chem Phys. 15, 7075-7086 (2013)

15. Duncan, J., Toor, H.: An experimental study of three component gas diffusion. AIChE J. 8, 38-41 (1962)

16. Gadau, S., Jüngel, A.: A three-dimensional mixed finite-element approximation of the semiconductor energy-transport equations. SIAM J. Sci Comput. 31, 1120-1140 (2008)

17. Galkin, V., Makashev, N.: Modification of the first approximation of the Chapman-Enskog method for a gas mixture. Fluid Dynam. 27, 590-596 (1993). Translated from em Izv. Ross. Akad. Nauk Mekh. Zhidk. Gaza 4 (1992), 178-185 (Russian)

18. Geiser, J.: Iterative solvers for the Maxwell-Stefan diffusion equations: methods and applications in plasma and particle transport. Cogent Math. 2, 1092913, 16. (2015)

19. Giovangigli, V.: Multicomponent Flow Modeling. Basel, Birkhäuser (1999)

20. Giovangigli, V., Massot, M.: The local Cauchy problem for multicomponent flows in full vibrational non-equilibrium. Math. Meth. Appl. Sci. 21, 1415-1439 (1998) 
21. Herberg, M., Meyries, M., Prüss, J., Wilke, M.: Reaction-diffusion systems of Maxwell-Stefan type with reversible mass-action kinetics. Nonlin. Anal. 159, 264-284 (2017)

22. Hutridurga, H., Salvarani, F.: Maxwell-Stefan diffusion asymptotics for gas mixtures in nonisothermal setting. Nonlin. Anal. 159, 285-297 (2017)

23. Hutridurga, H., Salvarani, F.: Existence and uniqueness analysis of a non-isothermal cross-diffusion system of Maxwell-Stefan type. Appl. Math. Lett. 75, 108-113 (2018)

24. Jüngel, A.: The boundedness-by-entropy method for cross-diffusion systems. Nonlinearity 28, 19632001 (2015)

25. Jüngel, A., Stelzer, I.: Entropy structure of a cross-diffusion tumor-growth model. Math. Models Meth. Appl. Sci. 22, 1250009, 26 (2012)

26. Jüngel, A., Stelzer, I.: Existence analysis of Maxwell-Stefan systems for multicomponent mixtures. SIAM J. Math Anal. 45, 2421-2440 (2013)

27. Leonardia, E., Angeli, C.: On the Maxwell-Stefan approach to diffusion: a general resolution in the transient regime for one-dimensional systems. J. Phys. Chem. B 114, 151-164 (2010)

28. Loos, J.-P., Verheijen, P., Moulin, J.: Numerical simulation of the generalized Maxwell-Stefan model for multicomponent diffusion in microporous sorbents. Collect. Czech. Chem Commun. 57, 687-697 (1992)

29. Marion, M., Temam, R.: Global existence for fully nonlinear reaction-diffusion systems describing multicomponent reactive flows. J. Math Pures Appl. 104, 102-138 (2015)

30. Maxwell, C.: On the dynamical theory of gases. Phil. Trans. Roy. Soc. Lond. 157, 49-88 (1866)

31. McLeod, M., Bourgault, Y.: Mixed finite element methods for addressing multi-species diffusion using the Maxwell-Stefan equations. Comput. Meth. Appl. Mech Engrg. 279, 515-535 (2014)

32. Nernst, W.: Die elektromotorische Wirksamkeit der Ionen. Z. Phys. Chem. 4, 129-181 (1889)

33. Peerenboom, K., van Dijk, J., Boonkkamp, J., Liu, L., Goedheer, W., van der Mullen, J.: Mass conservative finite volume discretization of the continuity equations in multi-component mixtures. J. Comput. Phys. 230, 3525-3537 (2011)

34. Planck, M.: Über die Potentialdifferenz zwischen zwei verdünnten Lösungen binärer Electrolyte. Ann. Phys. 276, 561-576 (1890)

35. Psaltis, S., Farrell, T.: Comparing charge transport predictions for a ternary electrolyte using the Maxwell-Stefan and Nernst-Planck equations. J. Electrochem. Soc. 158, A33-A42 (2011)

36. Salvarani, F., Soares, J.: On the relaxation of the Maxwell-Stefan system to linear diffusion. Appl. Math. Lett. 85, 15-21 (2018)

37. Stefan, J.: Über das Gleichgewicht und Bewegung, insbesondere die Diffusion von Gasgemengen. Sitzungsberichte Kaiserl. Akad. Wiss. Wien 63, 63-124 (1871) 